\title{
Synthesis of novel steroidal 16-spiroisoxazolines by 1,3-dipolar cycloaddition, and an evaluation of their antiproliferative activities in vitro
}

\author{
Éva Frank - Dóra Kovács - Gyula Schneider • \\ János Wölfling • Tibor Bartók • István Zupkó
}

Received: 20 November 2013 / Accepted: 7 March 2014

(C) Springer International Publishing Switzerland 2014

\begin{abstract}
Efficient synthesis of novel 16-spiroisoxazolines in the androst-5-ene series was carried out by 1,3-dipolar cycloadditions of different aryl nitrile oxides to $3 \beta$-acetoxy16-methylene-androst-5-en-17-one. During the intermolecular ring closures, the attack of the $\mathrm{O}$ terminus of the nitrile oxide dipole from the $\alpha$ side on C-16 predominated for steric reasons permitting the reactions to occur in a regio- and stereoselective manner. The minor isomers in which the angular methyl group on $\mathrm{C}-13$ and the $\mathrm{O}$ atom of the isoxazoline heteroring were in the $\beta, \beta$-cis orientation were obtained in a yield of only $\sim 10 \%$. Moreover, the conversions were influenced to a certain extent by the substituents on the aromatic moiety of the 1,3-dipoles. The stereostructures of the related diastereomers were confirmed by 2D NMR methods. Deacetylation of the primarily formed main products resulted in the corresponding $3 \beta-\mathrm{OH}$ analogs, which were further reduced to furnish $3 \beta, 17 \beta$-diols. All of the synthetized compounds were subjected to in vitro pharmacological studies in order to investigate their antiproliferative
\end{abstract}

Electronic supplementary material The online version of this article (doi:10.1007/s11030-014-9516-8) contains supplementary material, which is available to authorized users.

É. Frank $(\varangle) \cdot$ D. Kovács · G. Schneider · J. Wölfling

Department of Organic Chemistry, University of Szeged,

Dóm tér 8, Szeged 6720, Hungary

e-mail: frank@chem.u-szeged.hu

T. Bartók

Faculty of Engineering, University of Szeged, Moszkvai krt. 5-7,

Szeged 6725, Hungary

T. Bartók

Fumizol Ltd., Moszkvai krt. 5-7, Szeged 6725, Hungary

I. Zupkó

Department of Pharmacodynamics and Biopharmacy,

University of Szeged, Eötvös u. 6, Szeged 6720, Hungary effects on three malignant human adherent cell lines (HeLa, MCF7, and A431).

Keywords Steroid · Nitrile oxide - Cycloaddition . 16-Spiroisoxazolines $\cdot$ Stereoselective synthesis . Antiproliferative activity

\section{Introduction}

Steroids, an important class of naturally occurring regulatory molecules, are well known for their wide range of biological activities and have gained extensive application in the treatment of different diseases and in the improvement of physical and growth performance. Chemical modifications of the steroid nucleus, either by the introduction of heterocyclic moieties or by the replacement of one or more carbon atoms by a heteroatom, thereby giving rise to marked changes in the original bioactivity, have received considerable attention in recent years [1-3]. Considerable synthetic efforts have been devoted to the search for more active compounds untinged by unwanted or toxic side effects and to the recognition of the stereostructural features required for specific receptor binding and therefore selective pharmacological action. Consequently, both structure-based drug design and a more random search for effective derivatives appear to be fruitful routes in the quest for novel steroid-based medicinal agents. The formation of heterocyclic building blocks on the sterane core may alter both the pharmacokinetic and the pharmacodynamic properties of the parent compound, leading to hydrolysis-resistant derivatives with longer half-lives and/or to a better fit to the corresponding target through additional interactions made possible by the presence of a hetero ring. Moreover, the hydrophobic steroid scaffold can facili- 
tate the transportation of the introduced heterocycle through biological membranes.

Among the members of the large steroid family, spirosteroids represent an important class of compounds that are relatively widely found in nature, such as spirostanes and spirosolanes, which include a spiroacetal or spiroaminoacetal moiety and display significant biological effects. The glycoalkaloids solasonine and tomatine, extracted from different plant species, have demonstrated to exert strong antiproliferative effects on various human cancer cell lines of diverse origins $[4,5]$, while the synthetic spiro-type hybrid of estrone and talaromycin B has also been reported to exhibit cytotoxic activity [6,7]. In general, the spiro functionality, in which two rings are connected through merely one carbon atom, is a recurring structural motif in a number of natural products with noteworthy biological activities [8]. For example, coerulescine, horfiline, and elacomine exhibit antitumor effects, whereas rynchophylline and corynoxeine are used in traditional Chinese medicine for the treatment of neurological and cardiovascular diseases $[9,10]$.

The most investigated semi-synthetic spirosteroids are those containing a spiro heteroring at $\mathrm{C}-17$, but much less has been published regarding the synthesis of $\mathrm{C}-16$ spiroheterocyclic compounds [11]. Modifications involving the extant 17-keto functional group or the nearby position of the steroid core with the introduction of a bulky heterocyclic moiety can alter the primary stereostructure of the molecule, which may lead to a change in the substrate-receptor interaction and also greatly affect the biological properties. For this purpose, a number of different heterocyclic systems have been incorporated into the sterane skeleton in a spiro-connected manner, particulary, oxazolidinones [12], pyrazolines [13], pyrrolidines [14], dioxaphosphorinanes [15,16], oxazaphospholes [17], and oxathiaphospholanes [18]. The chemistry of steroidal spiroisoxazolines, however, has not been well investigated, although several 3- and 17-spiro derivatives have been synthetized to date from the corresponding methylene derivatives [19].

The established role of 2-isoxazolines as valuable intermediates in organic synthesis is attributed to their capacity to mask other functionalities within a stable form that allows further substitution of the ring [20]. $\alpha, \beta$-Unsaturated ketones [21], $\beta$-hydroxycarbonyl compounds [22], and 1,3aminoalcohols [23] are the most important structural units available from 2-isoxazolines by reductive cleavage of the hetero ring. Although the isoxazoline building block appears to be a rare functionality both in secondary metabolites found in nature [20,24] and among marketed pharmaceutical agents [25], several synthetic derivatives have been reported to exhibit valuable biological activities $[25,26]$. Several methods have been devised to construct such bio-important compounds, where the 1,3-dipolar cycloaddition of nitrile oxides to an unsaturated substrate has been widely investigated [19].
Nitrile oxides are generally obtained in situ from their relatively stable hydroximidoyl chloride precursors by dehydrohalogenation with a base [27]. In the absence, and even in the presence of the dipolarophile, nitrile oxides often rearrange to form an isocyanate at higher temperature or tend to dimerize to produce furoxan at room temperature, depending on their structure, and these side reactions can reduce the yields of the desired cycloadducts [28]. The Huisgen-type concerted reaction often leads to a regioisomeric isoxazoline mixture and needs elevated temperature and/or a prolonged reaction time for sufficient conversion.

As a continuation of our work for the construction of heterocyclic steroids possessing cell-growth inhibitory effect [29-32], we decided to prepare novel 16-spiroisoxazolinyl androst-5-ene derivatives from an $\alpha, \beta$-unsaturated steroidal 17-ketone via 1,3-dipolar cycloaddition. Our goal was to investigate the regio- and stereoselectivity of the process and the influence of steric and electronic factors on the ringclosure reactions. Determination of the stereostructures of the spiro compounds was also an aim of the present study. Moreover, all the synthetized derivatives were screened in vitro for their activities against a panel of three human adherent cancer cell lines (HeLa, MCF7, and A431).

\section{Results and discussion}

\section{Chemistry}

For the transformations, the starting material was $3 \beta$ acetoxy-16-methyleneandrost-5-en-17-one (2) [33], which is readily available from the 16-hydroxymethylene precursor 1 in the presence of an excess of formaldehyde and potassium carbonate via a formal mixed Cannizzaro reaction [34] and subsequent acetylation (Scheme 1). The presence of the exo-methylene group suggested the higher reactivity of the dipolarophile as compared to an endo-located double bond, and improved regioselectivity was expected in view of the monosubstituted character of the alkene moiety [27]. Moreover, conjugation with a $\mathrm{C}=\mathrm{O}$ bond has been demonstrated to have a strong driving effect on the reactivity of such alkenes [35]. Aromatic hydroximidoyl chlorides (5ae), as relatively stable precursors of nitrile oxide 1,3-dipoles (6a-e) [36], were synthetized in a two-step process by the condensation of benzaldehyde (3a) or its $p$-substituted derivatives (3b-e) with hydroxylamine hydrochloride in alkaline medium and subsequent chlorination of the aldoxime $\mathbf{4 a - e}$ with $N$-chlorosuccinimide (NCS) [37]. Nitrile oxides (6-e) can be generated in situ from $\mathbf{5 a - e}$ by dehydrochlorination with a base.

Preliminary ring-closure reactions on 2 with benzonitrile oxide 6a were first carried out to find the optimal reaction conditions (Scheme 1). 16-Methylene-17- 


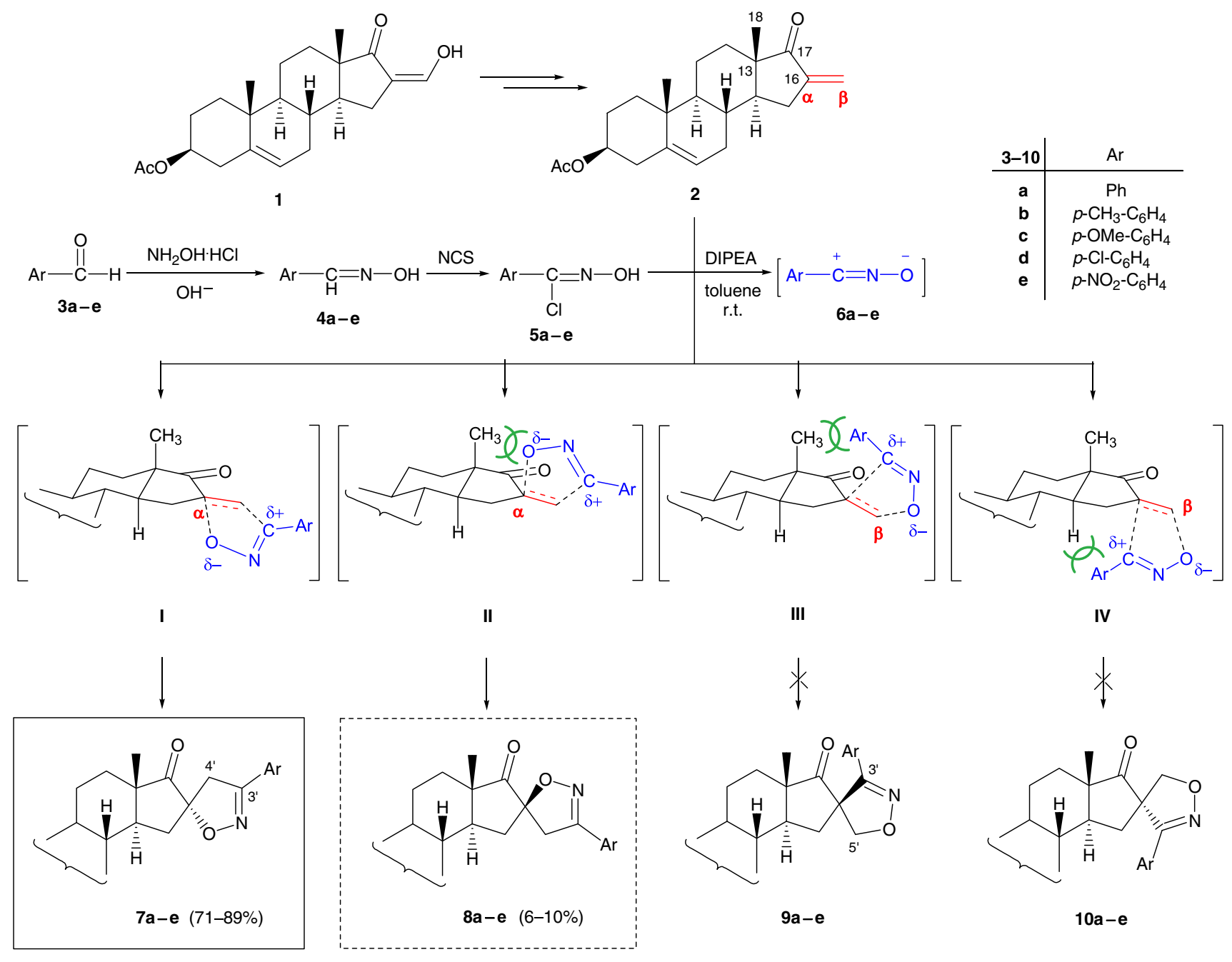

Scheme 1 Regio- and stereoselective formation of steroidal 16-spiroisoxazolines

ketosteroid (2) and $N$-hydroxybenzenecarboximidoyl chloride (5a) were dissolved in toluene and 3 equivalents of $N, N$ diisopropylethylamine (DIPEA) were added. Since unhindered 1,3-dipoles easily tend to dimerize to furoxanes, which can reduce their quantity available for cycloaddition [38], DIPEA was finally added to the solutions in order to avoid the formation of these unwanted by-products. After stirring of the mixtures for $2 \mathrm{~h}$ at room temperature, complete conversion was indicated by TLC, and two products (7a and $\mathbf{8 a}$ ) were obtained, in yields of 75 and $10 \%$, respectively, after chromatographic purification. Although the rate of the transformation could be enhanced by refluxing the solution, and the reaction was then completed within $1 \mathrm{~h}$, the application of milder conditions proved to be more favorable for further experiments in order to avoid the rearrangement of other dipoles to isocyanates at the elevated temperature. Similar intermolecular ring closures of $\mathbf{2}$ with different benzonitrile oxides $(\mathbf{6 b}-\mathbf{e})$, obtained from the appropriate aryl aldehydes $(\mathbf{3 b}-\mathbf{e})$ by the general protocol, were then carried out lead- ing to novel 16-spiroisoxazolines (7b-e and $\mathbf{8 b}-\mathbf{d}$ ) in good yields (Scheme 1). The formation of the possible $E$ and $Z$ isomers of both the aldoximes $(\mathbf{4 a}-\mathbf{e})$ and their chlorinated analogs $(\mathbf{5} \mathbf{a}-\mathbf{e})$ was detected by TLC; however, the unpurified hydroximidoyl chlorides $(\mathbf{5 a}-\mathbf{e})$ were applied for the subsequent cycloaddition reactions.

In principle, the construction of four isoxazolines (7-10) can be conceived in the ring-closure reactions of $\mathbf{2}$ with aromatic nitrile oxides (6a-e), as depicted in Scheme 1. The orientation of the 1,3-dipole relative to the double bond of the dipolarophile can be of two kinds: the negatively charged $\mathrm{O}$ terminus may interact with either the $\alpha$-or the $\beta$-carbon of the 16-methylene group of $\mathbf{2}$, and the attack can occur from above ( $\beta$ side) or from underneath ( $\alpha$ side) the general plane of the sterane molecule. Two regioisomeric pairs $(\mathbf{7}, \mathbf{8}$ and $\mathbf{9}$, 10), each involving two diastereomers, may therefore exist as concerns the newly formed stereogenic center on C-16, though only $\mathbf{7}$ and $\mathbf{8}$ (at least in most cases) were effectively obtained during the cycloadditions. The formation of regioi- 
Table 1 Cycloaddition products of 2 with different aromatic nitrile oxides $(\mathbf{6} \mathbf{a}-\mathbf{e})$

${ }^{a}$ Yields (\%) after purification by column chromatography are given in parenthesis

\begin{tabular}{lllll}
\hline Entry & $\mathrm{Ar}-\stackrel{+}{\mathrm{C}}=\mathrm{N}-\overline{\mathrm{C}}$ & $\mathrm{Ar}$ & Products $^{a}$ & Overall yields (\%) \\
\hline 1 & $\mathbf{6 a}$ & $\mathrm{Ph}$ & $\mathbf{7 a}(75)+\mathbf{8 a}(10)$ & 85 \\
2 & $\mathbf{6 b}$ & $p-\mathrm{CH}_{3}-\mathrm{C}_{6} \mathrm{H}_{4}$ & $\mathbf{7 b}(82)+\mathbf{8 b}(9)$ & 91 \\
3 & $\mathbf{6 c}$ & $p-\mathrm{OMe}-\mathrm{C}_{6} \mathrm{H}_{4}$ & $\mathbf{7 c}(89)+\mathbf{8 c}(6)$ & 95 \\
4 & $\mathbf{6 d}$ & $p-\mathrm{Cl}_{-}-\mathrm{C}_{6} \mathrm{H}_{4}$ & $\mathbf{7 d}(72)+\mathbf{8 d}(8)$ & 80 \\
5 & $\mathbf{6 e}$ & $p-\mathrm{NO}_{2}-\mathrm{C}_{6} \mathrm{H}_{4}$ & $\mathbf{7 e}(71)$ & 71 \\
\hline
\end{tabular}

somers (9 and 10) in which the $\mathrm{O}$ terminus is attached to the $\beta$-carbon of the dipolarophile is considered to be hampered by steric repulsions between the bulky aromatic ring of the nitrile oxide and the steroid portion. The attack of the anionic pole of the nitrile oxide from the $\beta$ side is also unfavorable due to the $\mathrm{C}-18$ angular methyl group with the same spatial orientation, although it does occur to a certain extent, leading to $\mathbf{8}$ as a minor by-product. With regard to the presumed transition states (I-IV), the most facilitated isomer is undoubtedly 7 , in which the $\mathrm{C}-\mathrm{O}$ bond of the heteroring is located in the $\alpha$ position opposite the methyl group on $\mathrm{C}-13$. Consequently, both the regio- and the stereoselectivity of the process are influenced by steric factors, in good agreement with earlier observations that the electronic character of the dipolarophile has only a minor effect on such reactions [39].

The overall yields of the epimeric products were affected by the electronic character of the substituents on the aryl moiety of the nitrile oxide $\mathbf{6 b}-\mathbf{e}$ (Table 1 ). The electron-donating $\mathrm{CH}_{3}$ and $\mathrm{OMe}$ groups in $\mathbf{6 b}$ and $\mathbf{6 c}$ (Table 1, entries 2 and 3 ) facilitated the cycloaddition to $\mathbf{2}$ due to the lower propensity of these dipoles to dimerize to furoxanes, while the presence of the electron-withdrawing $\mathrm{Cl}$ and $\mathrm{NO}_{2}$ substituents on the aromatic ring in $\mathbf{6} \mathbf{d}$ and $\mathbf{6 e}$ decreased the yields of the corresponding cycloadducts (7d and 8d, or 7e) (entries 4 and 5). The lowest conversion was found to occur for the reaction of 2 with $p$-nitrobenzonitrile oxide $\mathbf{6 e}$, which resulted in a single diastereomer (7e) in a yield of $71 \%$.

The ${ }^{13} \mathrm{C}$ NMR spectra recorded for $7 \mathbf{a}-\mathbf{e}$ and $\mathbf{8 a - d}$ confirmed the regioselectivity of the process, as the quaternary carbon signal of C-16 appeared at around 89 ppm, revealing the presence of an $\mathrm{O}$ atom adjacent to this carbon. In the other regioisomeric pairs (9 and 10), C-16 is next to C-5' of the isoxazolidine ring and its upfield shift would therefore be predicted. The stereostructures of the related epimers were established with the aid of homonuclear 2D NMR (COSY and NOESY) and heteronuclear 2D NMR (HSQC and $\mathrm{HMBC}$ ) measurements. The two diastereotopic protons of the C-4' methylene group appear as two doublets at 3.25 and $3.63 \mathrm{ppm}$ for $\mathbf{7 b}\left({ }^{2} J_{\mathrm{H}, \mathrm{C}, \mathrm{H}}=-16.5 \mathrm{~Hz}\right)$, and at 3.13 and $3.66 \mathrm{ppm}$ for $\mathbf{8 b}\left({ }^{2} J_{\mathrm{H}, \mathrm{C}, \mathrm{H}}=-16.4 \mathrm{~Hz}\right.$ ), (Schemes 2 and 3 ). The NOESY correlations revealed that the C-16 configuration is $S$ in $\mathbf{7 b}$ as both signals of the $4^{\prime}$-protons showed cross-peaks with the $\mathrm{C}-18$ methyl protons, while one of the doublets correlated with $15 \beta-\mathrm{H}$ (Scheme 2). The NOESY experiment on cycloadduct $\mathbf{8 b}$, however, supported the spatial vicinity of $15 \alpha-\mathrm{H}$ with one of the 4'-protons, showing a cross-peak between their signals, confirming the $R$ configuration of $\mathrm{C}-16$ in this case (Scheme 3 ).

For the enlargement of the compound library suitable for pharmacological studies and in the hope of finding structureactivity relationships, further derivatives of the main products $(\mathbf{7 a - e})$ were synthetized by simple deacetylations to furnish the corresponding $3 \beta$-hydroxy analogs (11a-e). Furthermore, $3 \beta, 17 \beta$-diols (12a-e) were obtained by stereoselective reduction of 11a-e (Scheme 4).

\section{Pharmacology}

Since a number of compounds of spiroisoxazoline type have been reported to exert noteworthy antiproliferative activities [40-42] and some steroidal derivatives containing similar heterocyclic moieties have also been demonstrated to inhibit cell proliferation $[26,29,32]$, the newly synthetized isoxazolines (7a-e, 8a-d, 11a-e, 12a-e) were subjected to in vitro pharmacological studies of their cytotoxic effects on three malignant human adherent cell lines, HeLa, MCF7, and A431 (Table 2). Their antiproliferative activities were determined by a microplate-based MTT colorimetric assay [43], in comparison with cisplatin as reference agent. The cell-proliferation inhibitory potencies, expressed as growth inhibition and/or $\mathrm{IC}_{50}$ values, revealed that several of the investigated compounds exhibited marked effects on cell proliferation, especially at $30 \mu \mathrm{M}$.

As concerns the structure-activity relationships, the configuration at $\mathrm{C}-16$ of the newly synthetized molecules seems to be the structural feature that mainly determines the antiproliferative properties, since $\mathbf{7 a - d}$ proved to be more potent than their epimeric counterparts 8a-d. Substitution of the aromatic ring on the isoxazoline moiety tended to increase the antiproliferative capacity in $\mathbf{7 b}-\mathbf{d}$, while the $p$-nitro group on the phenyl ring in $7 \mathbf{e}$ did not have a great impact on the overall efficacy as compared with 7a. Although the keto function at position 17 of the sterane skeleton is generally favorable, this part of the molecule and also the nature of the 
Scheme 2 Partial NOESY spectrum and $3 \mathrm{D}$ representation of main product $\mathbf{7 b}$
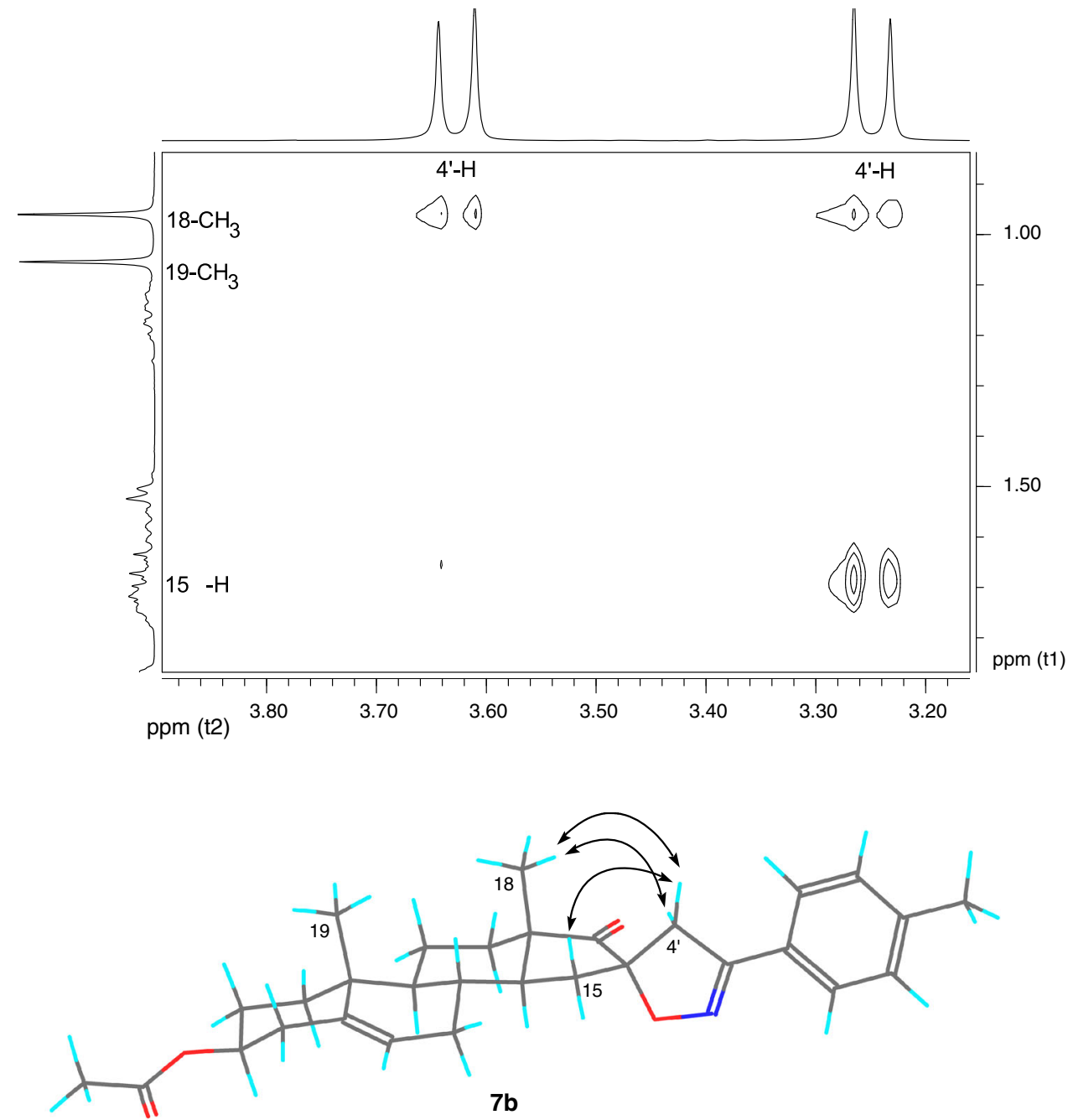

substituent $\mathrm{OAc}$ or $\mathrm{OH}$ at $\mathrm{C}-3$ did not have a crucial effect on the overall antiproliferative activities. The $\mathrm{IC}_{50}$ values of the two most potent agents, $\mathbf{7 d}$ and $\mathbf{1 1 d}$, both containing a $p$-chloro-phenyl-substituted isoxazoline building block with an $S$ configuration on C-16, were lower than or comparable to those of the reference agent cisplatin.

\section{Conclusions}

In summary, novel types of spiroisoxazolines in the $\Delta^{5}$ androstene series were prepared from a 16-methylene-17ketosteroid with different aromatic nitrile oxides via intermolecular 1,3-dipolar cycloaddition. The ring-closure reactions occurred under mild reaction conditions to afford the heterocyclic products regioselectively and stereoselectively in good to excellent yields. The conversions were observed to increase by the presence of electron-donating groups on the aromatic ring of the nitrile oxide in consequence of the lower tendency of these dipoles to undergo dimerization. The library of compounds was expanded by further deacetylation reactions and subsequent reductions. The cytotoxic efficacy of all compounds was investigated in vitro on three cancer cell lines, and several of the structurally related derivatives were found to have a marked effect on cell division. The pharmacological activities depended mainly on the stereochemistry and functionalization of the incorporated heterocycle rather than on the nature of the substituents on C-3 and/or C17 of the sterane core. Although only two derivatives of the currently tested agents proved to be specifically potent, the results indicate that steroidal spiroisoxazolines may deserve further attention not only from a synthetic but also from a pharmacological point of view.

\section{Experimental}

Melting points ( $\mathrm{mp}$ ) were determined on a SMS Optimelt digital apparatus. Elemental analysis data were obtained with a Perkin Elmer CHN analyzer model 2400. NMR spectra were 
Scheme 3 Partial NOESY spectrum and 3D representation of by-product $\mathbf{8 b}$
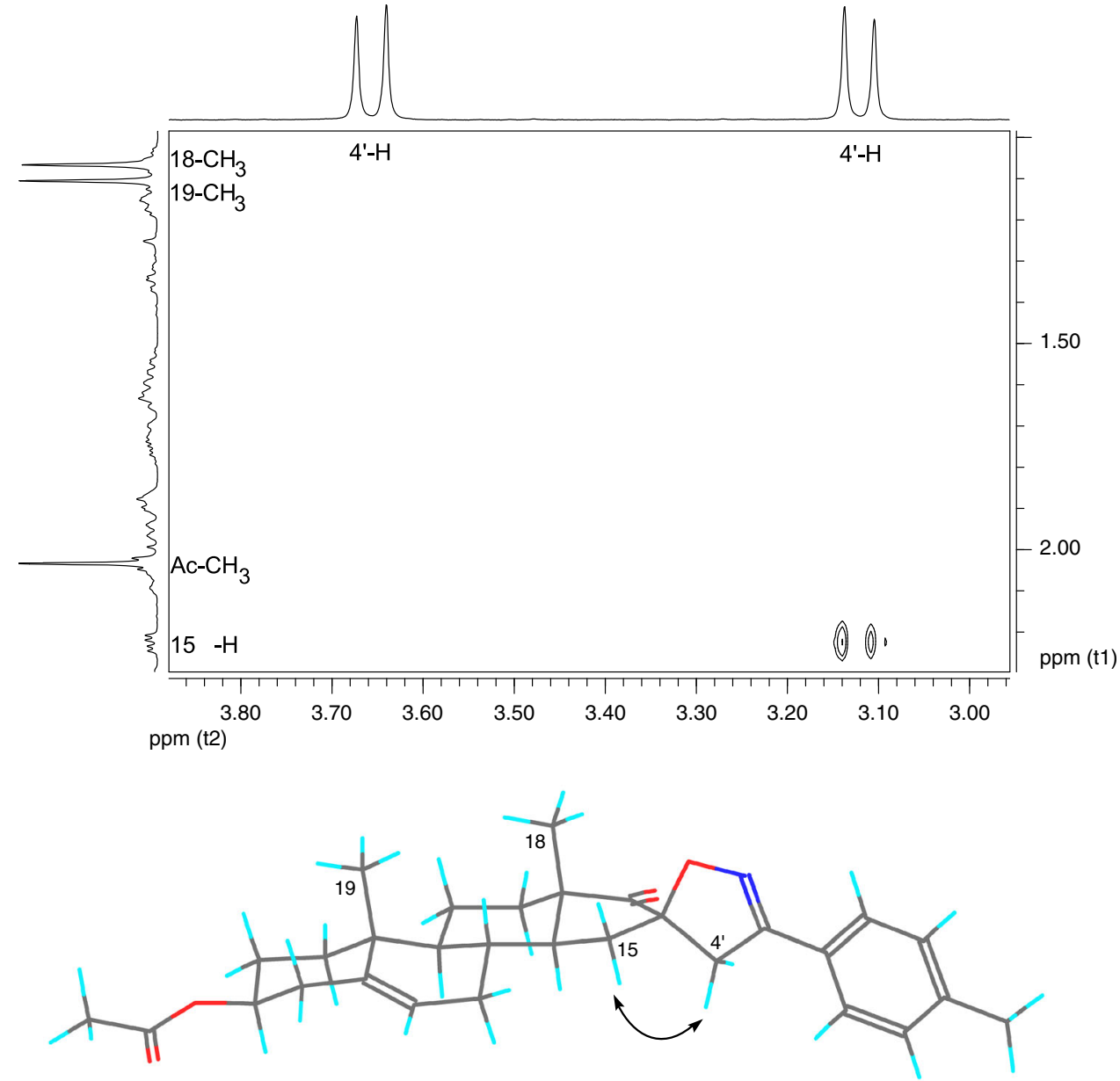

$8 b$

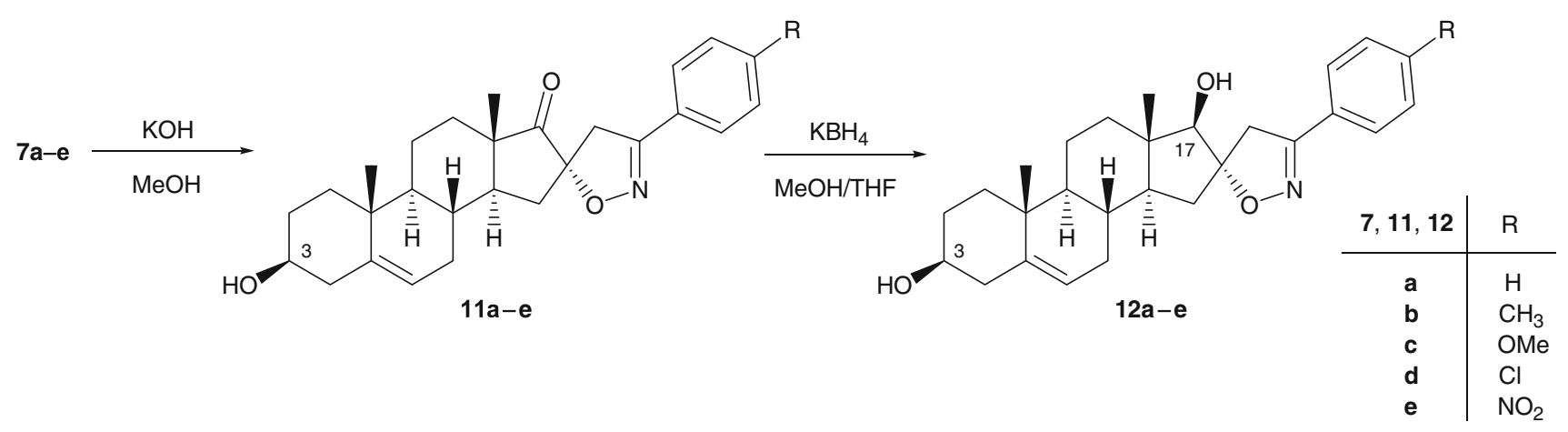

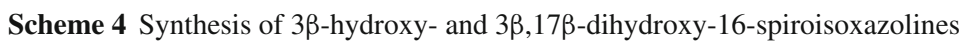

recorded at room temperature on a Bruker DRX 500 instrument. Chemical shifts are reported in ppm ( $\delta$ scale $)$ and coupling constants $(J)$ in Hz. For the determination of multiplicities, the $J$-MOD pulse sequence was used. Automated flow injection analyses were performed by using an HPLC/MSD system. The system comprised an Agilent 1100 micro vacuum degasser, a quaternary pump, a micro-well plate autoinjector, and a 1946A MSD equipped with an electrospray ion source (ESI) operated in positive ionization mode. The ESI parameters were nebulizing gas $\mathrm{N}_{2}$, at $35 \mathrm{psi}$; drying gas $\mathrm{N}_{2}$, at $350{ }^{\circ} \mathrm{C}$ and $12 \mathrm{~L} / \mathrm{min}$; capillary voltage ( $\left.V_{\text {Cap }}\right) 3,000$ $\mathrm{V}$; and fragmentor voltage $70 \mathrm{~V}$. The MSD was operated in scan mode with the mass range $m / z$ 60-620. Samples $(0.2$ $\mu \mathrm{L})$ were injected with an automated needle wash directly into the solvent flow $(0.3 \mathrm{~mL} / \mathrm{min})$ of $\mathrm{MeCN} / \mathrm{H}_{2} \mathrm{O} 70: 30$ (v/v) supplemented with $0.1 \%$ formic acid. The system was 
Table 2 Cytotoxic activities of spiroisoxazolines in the androstene series

\begin{tabular}{|c|c|c|c|c|c|c|c|}
\hline Compd & Conc. $(\mu \mathrm{M})$ & $\begin{array}{l}\text { HeLa } \\
\text { Inhibition \% }( \pm \text { SEM })\end{array}$ & $\mathrm{IC}_{50}^{\mathrm{a}}(\mu \mathrm{M})$ & $\begin{array}{l}\text { MCF7 } \\
\text { Inhibition \% }( \pm \text { SEM })\end{array}$ & $\mathrm{IC}_{50}(\mu \mathrm{M})$ & $\begin{array}{l}\text { A431 } \\
\text { Inhibition \% }( \pm \text { SEM })\end{array}$ & $\mathrm{IC}_{50}(\mu \mathrm{M})$ \\
\hline \multirow[t]{2}{*}{$7 \mathbf{a}$} & 10 & $59.7( \pm 1.2)$ & $-{ }^{\mathrm{b}}$ & $53.5( \pm 1.1)$ & - & $42.1( \pm 1.1)$ & - \\
\hline & 30 & $81.5( \pm 1.9)$ & - & $68.1( \pm 1.0)$ & - & $48.0( \pm 1.1)$ & - \\
\hline \multirow[t]{2}{*}{$7 b$} & 10 & $82.5( \pm 0.6)$ & - & $84.7( \pm 0.5)$ & - & $65.9( \pm 0.7)$ & - \\
\hline & 30 & $94.3( \pm 1.3)$ & - & $94.0( \pm 0.8)$ & - & $83.3( \pm 0.5)$ & - \\
\hline \multirow[t]{2}{*}{$7 c$} & 10 & $61.4( \pm 1.2)$ & - & $66.0( \pm 1.1)$ & - & $34.2( \pm 2.2)$ & - \\
\hline & 30 & $86.7( \pm 0.7)$ & - & $74.3( \pm 0.6)$ & - & $58.2( \pm 1.2)$ & - \\
\hline \multirow[t]{2}{*}{$7 d$} & 10 & $97.7( \pm 0.2)$ & 7.46 & $92.0( \pm 1.0)$ & 8.07 & $98.1( \pm 0.5)$ & 3.42 \\
\hline & 30 & $98.5( \pm 0.1)$ & & $95.6( \pm 0.2)$ & & $98.1( \pm 0.4)$ & \\
\hline \multirow[t]{2}{*}{$7 e$} & 10 & $55.4( \pm 1.2)$ & - & $56.1( \pm 2.6)$ & - & $34.2( \pm 1.3)$ & - \\
\hline & 30 & $55.8( \pm 2.7)$ & - & $65.8( \pm 1.4)$ & - & $38.3( \pm 1.9)$ & - \\
\hline \multirow[t]{2}{*}{$8 \mathbf{a}$} & 10 & $<20^{\mathrm{c}}$ & - & $<20$ & - & $<20$ & - \\
\hline & 30 & $32.0( \pm 1.1)$ & - & $64.7( \pm 1.8)$ & - & $<20$ & - \\
\hline \multirow[t]{2}{*}{$8 b$} & 10 & $<20$ & - & $26.6( \pm 2.9)$ & - & $<20$ & - \\
\hline & 30 & $59.8( \pm 1.8)$ & - & $49.9( \pm 1.3)$ & - & $<20$ & - \\
\hline \multirow[t]{2}{*}{$8 c$} & 10 & $<20$ & - & $20.8( \pm 2.0)$ & - & $<20$ & - \\
\hline & 30 & $<20$ & - & $31.8( \pm 1.3)$ & - & $<20$ & - \\
\hline \multirow[t]{2}{*}{$8 d$} & 10 & $33.5( \pm 1.6)$ & - & $23.0( \pm 0.7)$ & - & $<20$ & - \\
\hline & 30 & $72.4( \pm 2.7)$ & - & $63.8( \pm 1.4)$ & - & $30.5( \pm 2.1)$ & - \\
\hline \multirow[t]{2}{*}{$11 \mathrm{a}$} & 10 & $<20$ & - & $35.2( \pm 1.2)$ & - & $<20$ & \\
\hline & 30 & $96.8( \pm 0.3)$ & - & $98.2( \pm 0.3)$ & - & $95.8( \pm 0.4)$ & \\
\hline \multirow[t]{2}{*}{$11 b$} & 10 & $60.3( \pm 0.6)$ & - & $51.5( \pm 1.7)$ & - & $58.0( \pm 1.2)$ & - \\
\hline & 30 & $82.5( \pm 1.0)$ & - & $88.5( \pm 0.7)$ & - & $76.1( \pm 0.9)$ & - \\
\hline \multirow[t]{2}{*}{$11 \mathrm{c}$} & 10 & $42.7( \pm 1.3)$ & - & $70.0( \pm 1.4)$ & - & $30.6( \pm 2.8)$ & \\
\hline & 30 & $82.3( \pm 1.8)$ & - & $82.8( \pm 1.4)$ & - & $41.4( \pm 0.7)$ & \\
\hline \multirow[t]{2}{*}{ 11d } & 10 & $88.6( \pm 2.7)$ & 7.39 & $92.9( \pm 0.3)$ & 7.13 & $67.3( \pm 2.9)$ & 4.20 \\
\hline & 30 & $98.3( \pm 0.2)$ & & $93.6( \pm 1.1)$ & & $98.2( \pm 0.1)$ & \\
\hline \multirow[t]{2}{*}{$11 e$} & 10 & $<20$ & - & $<20$ & - & $<20$ & - \\
\hline & 30 & $33.5( \pm 1.3)$ & - & $30.1( \pm 1.8)$ & - & $20.6( \pm 2.6)$ & - \\
\hline \multirow[t]{2}{*}{$12 \mathbf{a}$} & 10 & $<20$ & - & $<20$ & - & $<20$ & - \\
\hline & 30 & $97.6( \pm 0.1)$ & - & $95.9( \pm 1.4)$ & - & $95.6( \pm 0.3)$ & - \\
\hline \multirow[t]{2}{*}{$12 b$} & 10 & $36.4( \pm 1.3)$ & - & $<20$ & - & $<20$ & - \\
\hline & 30 & $96.4( \pm 0.4)$ & - & $98.6( \pm 0.1)$ & - & $96.7( \pm 0.5)$ & - \\
\hline \multirow[t]{2}{*}{$12 \mathrm{c}$} & 10 & $<20$ & - & $26.4( \pm 2.0)$ & - & $<20$ & - \\
\hline & 30 & $50.9( \pm 0.7)$ & - & $74.1( \pm 1.1)$ & - & $45.2( \pm 2.4)$ & - \\
\hline \multirow[t]{2}{*}{ 12d } & 10 & $50.7( \pm 0.7)$ & - & $32.4( \pm 2.8)$ & - & $44.1( \pm 0.8)$ & - \\
\hline & 30 & $57.7( \pm 1.1)$ & - & $32.6( \pm 1.4)$ & - & $46.7( \pm 0.6)$ & - \\
\hline \multirow[t]{2}{*}{$12 \mathrm{e}$} & 10 & $58.8( \pm 0.8)$ & - & $51.6( \pm 1.6)$ & - & $61.4( \pm 1.7)$ & - \\
\hline & 30 & $73.7( \pm 1.9)$ & - & $73.8( \pm 1.2)$ & - & $86.5( \pm 0.8)$ & - \\
\hline \multirow[t]{2}{*}{$\mathbf{C P}^{\mathrm{d}}$} & 10 & $42.6( \pm 2.3)$ & 12.43 & $53.0( \pm 2.3)$ & 9.63 & $88.6( \pm 0.5)$ & 2.84 \\
\hline & 30 & $99.9( \pm 0.3)$ & & $86.9( \pm 1.3)$ & & $90.2( \pm 1.8)$ & \\
\hline
\end{tabular}

${ }^{\mathrm{a}} \mathrm{IC}_{50}$ values were determined when the tested compound elicited at least $50 \%$ growth inhibition at $10 \mu \mathrm{M}$ against any of the cell lines used. The presented values are from two independent determinations with five parallel wells; standard deviation $<15 \%$

${ }^{\mathrm{b}}$ Not determined

${ }^{\mathrm{c}}$ Inhibition values $<20 \%$ are not presented

${ }^{\mathrm{d}}$ Cisplatin (reference compound) 
controlled by Agilent's LC/MSD Chemstation software. All solvents were distilled immediately prior to use. Reagents and materials were obtained from commercial suppliers and were used without purification. The reactions were monitored by TLC on Kieselgel-G (Merck Si $254 \mathrm{~F}$ ) layers $(0.25 \mathrm{~mm}$ thick); solvent systems (ss): (A) EtOAc/ $\mathrm{CH}_{2} \mathrm{Cl}_{2}(2: 98 \mathrm{v} / \mathrm{v})$, (B) $\mathrm{EtOAc} / \mathrm{CH}_{2} \mathrm{Cl}_{2}(10: 90 \mathrm{v} / \mathrm{v})$, (C) EtOAc/ $\mathrm{CH}_{2} \mathrm{Cl}_{2}(25: 75$ $\mathrm{v} / \mathrm{v})$. The spots were developed by spraying with $5 \%$ phosphomolybdic acid in $50 \%$ aqueous phosphoric acid. The $R_{f}$ values were determined for the spots observed by illumination at 254 and $365 \mathrm{~nm}$. Flash chromatography: Merck silica gel 60, 40-63 $\mu \mathrm{m}$.

General procedure for the synthesis of 16-spiroisoxazolyl derivatives ( 7 and $\mathbf{8}$ ) in the $\Delta^{5}$ androstene series

3ß-Acetoxy-16-methyleneandrost-5-en-17-one (2) (343 mg, $1.00 \mathrm{mmol}$ ) and the appropriate aromatic imidoyl chloride [36] (5a-e, $1.50 \mathrm{mmol})$ were dissolved in toluene $(15 \mathrm{~mL})$, and DIPEA $(0.52 \mathrm{~mL}, 3.00 \mathrm{mmol})$ was added dropwise to the reaction mixture, which was subsequently stirred at room temperature for $2 \mathrm{~h}$. The solvent was then evaporated off in vacuo and the resulting crude products were separated by column chromatography.

Synthesis of $3 \beta$-acetoxy-3'-phenyl-spiro [androst-5-ene16,5'-2'-isoxazolin]-17-one epimers (7a and $\mathbf{8 a})$

According to Sect. 4.1, N-hydroxybenzenecarboximidoyl chloride (5a, $233 \mathrm{mg}$ ) was used. After purification with $\mathrm{CH}_{2} \mathrm{Cl}_{2}$ as eluent, 7a (346 mg, $75 \%$ ) and 8a (46 mg, 10 $\%$ ) were obtained as white solids (sequence of elution: $\mathbf{8 a}>$ 7a).

7a: $\mathrm{mp} 245-248^{\circ} \mathrm{C}, R_{\mathrm{f}}=0.38(\mathrm{ss} \mathrm{A}) ;{ }^{1} \mathrm{H} \mathrm{NMR}\left(\mathrm{CDCl}_{3}\right.$, $500 \mathrm{MHz}): \delta_{\mathrm{H}} 0.97\left(\mathrm{~s}, 3 \mathrm{H}, 18-\mathrm{H}_{3}\right), 1.06\left(\mathrm{~s}, 3 \mathrm{H}, 19-\mathrm{H}_{3}\right), 1.14$ $(\mathrm{m}, 2 \mathrm{H}), 1.48-1.75$ (overlapping $\mathrm{m}, 8 \mathrm{H}$ ), 1.86-1.96 (overlapping m, 3H), 2.03 (s, 3H, Ac- $\left.\mathrm{CH}_{3}\right), 2.04(\mathrm{~m}, 1 \mathrm{H}), 2.35$ $(\mathrm{m}, 3 \mathrm{H}), 3.27\left(\mathrm{~d}, 1 \mathrm{H}, J=16.6 \mathrm{~Hz}\right.$, one of $\left.4^{\prime}-\mathrm{H}\right), 3.65(\mathrm{~d}, 1 \mathrm{H}$, $J=16.6 \mathrm{~Hz}$, the other $\left.4^{\prime}-\mathrm{H}\right), 4.60(\mathrm{~m}, 1 \mathrm{H}, 3-\mathrm{H}), 5.40(\mathrm{~m}, 1 \mathrm{H}$, 6-H), 7.38 (m, 3H, 3" $-\mathrm{H}, 4^{\prime \prime}-\mathrm{H}$ and $\left.5^{\prime \prime}-\mathrm{H}\right), 7.63$ (m, 2H, $2^{\prime \prime}-\mathrm{H}$ and $\left.6{ }^{\prime \prime}-\mathrm{H}\right) ;{ }^{13} \mathrm{C} \mathrm{NMR}\left(\mathrm{CDCl}_{3}, 125 \mathrm{MHz}\right): \delta_{\mathrm{C}} 14.3(\mathrm{C}-18)$, 19.3 (C-19), $20.2\left(\mathrm{CH}_{2}\right), 21.4\left(\mathrm{Ac}-\mathrm{CH}_{3}\right), 27.6\left(\mathrm{CH}_{2}\right), 30.7$ $\left(\mathrm{CH}_{2}\right), 31.0(\mathrm{CH}), 31.6\left(\mathrm{CH}_{2}\right), 36.7(\mathrm{C}-10), 36.8\left(\mathrm{CH}_{2}\right), 37.5$ $\left(\mathrm{CH}_{2}\right), 38.0\left(\mathrm{CH}_{2}\right), 44.0\left(\mathrm{CH}_{2}\right), 47.1(\mathrm{C}-13), 48.5(\mathrm{CH}), 49.9$ (CH), 73.6 (C-3), 88.7 (C-16), 121.7 (C-6), 126.8 (2C, C-3" and $\left.\mathrm{C}-5^{\prime \prime}\right), 128.7$ (2C, C-2 ${ }^{\prime \prime}$ and $\left.\mathrm{C}-6^{\prime \prime}\right), 129.0\left(\mathrm{C}-1^{\prime \prime}\right), 130.2$ $\left(\mathrm{C}-4^{\prime \prime}\right), 139.9(\mathrm{C}-5), 155.3\left(\mathrm{C}-3^{\prime \prime}\right), 170.4$ (Ac-CO), $216.2(\mathrm{C}-$ 17); ESI-MS $485[\mathrm{M}+\mathrm{Na}]^{+}$; Anal. Calcd. for $\mathrm{C}_{29} \mathrm{H}_{35} \mathrm{NO}_{4} \mathrm{C}$ 75.46; H 7.64. Found C 75.62; H 7.80.

8a: $\mathrm{mp} 187-190{ }^{\circ} \mathrm{C}, R_{\mathrm{f}}=0.44$ (ss A); ${ }^{1} \mathrm{H} \mathrm{NMR}\left(\mathrm{CDCl}_{3}\right.$, $500 \mathrm{MHz}): \delta_{\mathrm{H}} 1.05(\mathrm{~m}, 1 \mathrm{H}), 1.07\left(\mathrm{~s}, 3 \mathrm{H}, 19-\mathrm{H}_{3}\right), 1.11(\mathrm{~s}$, $\left.3 \mathrm{H}, 18-\mathrm{H}_{3}\right), 1.15(\mathrm{~m}, 2 \mathrm{H}), 1.34(\mathrm{~m}, 1 \mathrm{H}), 1.52-1.66(\mathrm{~m}, 3 \mathrm{H})$, $1.70-1.80(\mathrm{~m}, 2 \mathrm{H}), 1.88-1.98(\mathrm{~m}, 3 \mathrm{H}), 2.03$ (s, 3H, Ac- $\left.\mathrm{CH}_{3}\right)$, $2.04(\mathrm{~m}, 1 \mathrm{H}), 2.08(\mathrm{~m}, 1 \mathrm{H}), 2.24(\mathrm{dd}, 1 \mathrm{H}, J=13.1 \mathrm{~Hz}, J$ $=5.8 \mathrm{~Hz}), 2.35(\mathrm{~m}, 2 \mathrm{H}), 3.14(\mathrm{~d}, 1 \mathrm{H}, J=16.4 \mathrm{~Hz}$, one of $\left.4^{\prime}-\mathrm{H}\right), 3.69\left(\mathrm{~d}, 1 \mathrm{H}, J=16.4 \mathrm{~Hz}\right.$, the other $\left.4^{\prime}-\mathrm{H}\right), 4.61(\mathrm{~m}, 1 \mathrm{H}$, 3-H), $5.39(\mathrm{~m}, 1 \mathrm{H}, 6-\mathrm{H}), 7.39\left(\mathrm{~m}, 3 \mathrm{H}, 3^{\prime \prime}-\mathrm{H}, 4^{\prime \prime}-\mathrm{H}\right.$ and $5^{\prime \prime}-$ $\mathrm{H}), 7.64\left(\mathrm{~m}, 2 \mathrm{H}, 2^{\prime \prime}-\mathrm{H}\right.$ and $\left.6^{\prime \prime}-\mathrm{H}\right) ;{ }^{13} \mathrm{C}-\mathrm{NMR}\left(\mathrm{CDCl}_{3}, 125\right.$ $\mathrm{MHz}): \delta_{\mathrm{C}} 14.3(\mathrm{C}-18), 19.3(\mathrm{C}-19), 20.1\left(\mathrm{CH}_{2}\right), 21.4(\mathrm{Ac}-$ $\left.\mathrm{CH}_{3}\right), 27.6\left(\mathrm{CH}_{2}\right), 30.8\left(\mathrm{CH}_{2}\right), 30.9(\mathrm{CH}), 31.9\left(\mathrm{CH}_{2}\right), 36.8$ (C-10), $36.9\left(\mathrm{CH}_{2}\right), 38.0\left(2 \mathrm{C}, 2 \times \mathrm{CH}_{2}\right), 45.4\left(\mathrm{CH}_{2}\right), 46.4$ (CH), 46.8 (C-13), 50.2 (CH), 73.6 (C-3), 88.6 (C-16), 121.4 (C-6), $126.8\left(2 \mathrm{C}, \mathrm{C}-3^{\prime \prime}\right.$ and C-5"), 128.7 (2C, C-2" and C$\left.6^{\prime \prime}\right), 129.0\left(\mathrm{C}-1^{\prime \prime}\right), 130.2\left(\mathrm{C}-4^{\prime \prime}\right), 140.0(\mathrm{C}-5), 155.0\left(\mathrm{C}-3^{\prime}\right)$, 170.5 (Ac-CO), 215.4 (C-17); ESI-MS 485 [M+Na] ${ }^{+}$; Anal. Calcd. for $\mathrm{C}_{29} \mathrm{H}_{35} \mathrm{NO}_{4} \mathrm{C} 75.46 ; \mathrm{H}$ 7.64. Found $\mathrm{C} 75.60 ; \mathrm{H}$ 7.78 .

Synthesis of $3 \beta$-acetoxy- $3^{\prime}-4^{\prime \prime}$-tolyl-spiro[androst-5-ene16,5'-2'-isoxazolin]-17-one epimers (7b and $\mathbf{8 b}$ )

According to Sect. 4.1, N-hydroxy-4-methylbenzenecarboximidoyl chloride (5b, $254 \mathrm{mg}$ ) was used. After purification with EtOAc/ $\mathrm{CH}_{2} \mathrm{Cl}_{2}=2: 98$ as eluent, $7 \mathbf{b}(390 \mathrm{mg}$, $82 \%)$ and $\mathbf{8 b}(43 \mathrm{mg}, 9 \%)$ were obtained as white solids (sequence of elution: $\mathbf{8 b}>\mathbf{7 b}$ ).

7b: $\mathrm{mp} 240-242{ }^{\circ} \mathrm{C}, R_{f}=0.32$ (ss A); ${ }^{1} \mathrm{H} \mathrm{NMR}\left(\mathrm{CDCl}_{3}\right.$, $500 \mathrm{MHz}): \delta_{\mathrm{H}} 0.96\left(\mathrm{~s}, 3 \mathrm{H}, 18-\mathrm{H}_{3}\right), 1.05\left(\mathrm{~s}, 3 \mathrm{H}, 19-\mathrm{H}_{3}\right), 1.50$ (m, 2H), 1.48-1.77 (overlapping $\mathrm{m}, 8 \mathrm{H}), 1.88$ (m, 2H), 1.95 $(\mathrm{m}, 1 \mathrm{H}), 2.03\left(\mathrm{~s}, 3 \mathrm{H}, \mathrm{Ac}-\mathrm{CH}_{3}\right), 2.05(\mathrm{~m}, 1 \mathrm{H}), 2.34(\mathrm{~m}, 3 \mathrm{H})$, $2.37\left(\mathrm{~s}, 3 \mathrm{H}, 4^{\prime \prime}-\mathrm{CH}_{3}\right), 3.25\left(\mathrm{~d}, 1 \mathrm{H}, J=16.5 \mathrm{~Hz}\right.$, one of $\left.4^{\prime}-\mathrm{H}\right)$, $3.63\left(\mathrm{~d}, 1 \mathrm{H}, J=16.5 \mathrm{~Hz}\right.$, the other $\left.4^{\prime}-\mathrm{H}\right), 4.61(\mathrm{~m}, 1 \mathrm{H}, 3-\mathrm{H})$, $5.40(\mathrm{~m}, 1 \mathrm{H}, 6-\mathrm{H}), 7.19\left(\mathrm{~d}, 2 \mathrm{H}, J=8.0 \mathrm{~Hz}, 3^{\prime \prime}-\mathrm{H}\right.$ and $\left.5^{\prime \prime}-\mathrm{H}\right)$, $7.53\left(\mathrm{~d}, 2 \mathrm{H}, J=8.0 \mathrm{~Hz}, 2^{\prime \prime}-\mathrm{H}\right.$ and $\left.6{ }^{\prime \prime}-\mathrm{H}\right) ;{ }^{13} \mathrm{C} \mathrm{NMR}\left(\mathrm{CDCl}_{3}\right.$, $125 \mathrm{MHz}): \delta_{\mathrm{C}} 14.3(\mathrm{C}-18), 19.3(\mathrm{C}-19), 20.2\left(\mathrm{CH}_{2}\right), 21.4$ $\left(2 \mathrm{C}, 4^{\prime \prime}-\mathrm{CH}_{3}\right.$ and $\left.\mathrm{Ac}-\mathrm{CH}_{3}\right), 27.6\left(\mathrm{CH}_{2}\right), 30.6\left(\mathrm{CH}_{2}\right), 31.0$ $(\mathrm{CH}), 31.6\left(\mathrm{CH}_{2}\right), 36.7(\mathrm{C}-10), 36.8\left(\mathrm{CH}_{2}\right), 37.6\left(\mathrm{CH}_{2}\right), 38.0$ $\left(\mathrm{CH}_{2}\right), 44.2\left(\mathrm{CH}_{2}\right), 47.1(\mathrm{C}-13), 48.5(\mathrm{CH}), 49.9(\mathrm{CH}), 73.6$ (C-3), 88.5 (C-16), 121.7 (C-6), $126.2\left(\mathrm{C}-1^{\prime \prime}\right), 126.8$ (2C, C$2^{\prime \prime}$ and C- $\left.6^{\prime \prime}\right), 129.4\left(2 \mathrm{C}, \mathrm{C}-3^{\prime \prime}\right.$ and C-5" $), 139.8\left(\mathrm{C}-4^{\prime \prime}\right), 140.4$ (C-5), 155.3 (C-3'), 170.4 (Ac-CO), 216.4 (C-17); ESI-MS $477[\mathrm{M}+\mathrm{H}]^{+}$; Anal. Calcd. for $\mathrm{C}_{30} \mathrm{H}_{37} \mathrm{NO}_{4} \mathrm{C} 75.76 ; \mathrm{H} 7.84$. Found C 75.92; H 8.00.

8b: mp $193-195{ }^{\circ} \mathrm{C}, R_{\mathrm{f}}=0.51$ (ss A); ${ }^{1} \mathrm{H} \mathrm{NMR}\left(\mathrm{CDCl}_{3}\right.$, $500 \mathrm{MHz}): \delta_{\mathrm{H}} 1.05(\mathrm{~m}, 1 \mathrm{H}), 1.06\left(\mathrm{~s}, 3 \mathrm{H}, 19-\mathrm{H}_{3}\right), 1.11(\mathrm{~s}$, $\left.3 \mathrm{H}, 18-\mathrm{H}_{3}\right), 1.15(\mathrm{~m}, 2 \mathrm{H}), 1.34(\mathrm{~m}, 1 \mathrm{H}), 1.51-1.66(\mathrm{~m}, 3 \mathrm{H})$, 1.70-1.79 (m, 2H), 1.87-1.99 (m, 3H), $2.02(\mathrm{~m}, 1 \mathrm{H}), 2.03$ $\left(\mathrm{s}, 3 \mathrm{H}, \mathrm{Ac}_{-} \mathrm{CH}_{3}\right), 2.08(\mathrm{~m}, 1 \mathrm{H}), 2.23(\mathrm{dd}, 1 \mathrm{H}, J=13.1 \mathrm{~Hz}$, $J=5.7 \mathrm{~Hz}$ ), $2.35(\mathrm{~m}, 2 \mathrm{H}), 2.37\left(\mathrm{~s}, 3 \mathrm{H}, 4^{\prime \prime}-\mathrm{CH}_{3}\right), 3.13$ (d, $1 \mathrm{H}, J=16.4 \mathrm{~Hz}$, one of $\left.4^{\prime}-\mathrm{H}\right), 3.66(\mathrm{~d}, 1 \mathrm{H}, J=16.4 \mathrm{~Hz}$, the other $\left.4^{\prime}-\mathrm{H}\right), 4.61(\mathrm{~m}, 1 \mathrm{H}, 3-\mathrm{H}), 5.39(\mathrm{~m}, 1 \mathrm{H}, 6-\mathrm{H}), 7.19$ (d, $2 \mathrm{H}, J=8.0 \mathrm{~Hz}, 3^{\prime \prime}-\mathrm{H}$ and $\left.5^{\prime \prime}-\mathrm{H}\right), 7.53(\mathrm{~d}, 2 \mathrm{H}, J=8.0 \mathrm{~Hz}$, $2^{\prime \prime}-\mathrm{H}$ and $\left.6{ }^{\prime \prime}-\mathrm{H}\right) ;{ }^{13} \mathrm{C} \mathrm{NMR}\left(\mathrm{CDCl}_{3}, 125 \mathrm{MHz}\right): \delta_{\mathrm{C}} 130.0$ (C-18), 19.3 (C-19), $20.1\left(\mathrm{CH}_{2}\right), 21.4\left(2 \mathrm{C}, 4^{\prime \prime}-\mathrm{CH}_{3}\right.$ and Ac$\left.\mathrm{CH}_{3}\right), 27.6\left(\mathrm{CH}_{2}\right), 30.8\left(\mathrm{CH}_{2}\right), 30.9(\mathrm{CH}), 31.8\left(\mathrm{CH}_{2}\right), 36.8$ (C-10), $36.9\left(\mathrm{CH}_{2}\right), 38.0\left(2 \mathrm{C}, 2 \times \mathrm{CH}_{2}\right), 45.6\left(\mathrm{CH}_{2}\right), 46.4$ 
(CH), 46.8 (C-13), $50.2(\mathrm{CH}), 73.6(\mathrm{C}-3), 88.5(\mathrm{C}-16), 121.4$ (C-6), $126.1\left(\mathrm{C}-1^{\prime \prime}\right), 126.8$ (2C, C-2" and C-6"), 129.4 (2C, $\mathrm{C}-3^{\prime \prime}$ and C-5"), 140.0 (C-4"), 140.5 (C-5), 154.9 (C-3'), 170.5 (Ac-CO), 215.5 (C-17); ESI-MS 477 [M+H] ${ }^{+}$; Anal. Calcd. for $\mathrm{C}_{30} \mathrm{H}_{37} \mathrm{NO}_{4} \mathrm{C} 75.76 ; \mathrm{H}$ 7.84. Found $\mathrm{C} 75.62 ; \mathrm{H}$ 7.94.

Synthesis of $3 \beta$-acetoxy-3'-4"-methoxyphenyl-spiro [androst-5-ene-16,5'-2'-isoxazolin]-17-one epimers ( $\mathbf{7} \mathbf{c}$ and 8c)

According to Sect. 4.1, $N$-hydroxy-4-methoxybenzenecarboximidoyl chloride (5c, $279 \mathrm{mg}$ ) was used. After purification with $\mathrm{CH}_{2} \mathrm{Cl}_{2}$ as eluent, 7c (438 mg, $89 \%$ ) and 8c (29 $\mathrm{mg}, 6 \%$ ) were obtained as white solids (sequence of elution: $7 \mathrm{c}>8 \mathrm{c})$.

7c: $\mathrm{mp} 248-250{ }^{\circ} \mathrm{C}, R_{\mathrm{f}}=0.26(\mathrm{ss} \mathrm{A}) ;{ }^{1} \mathrm{H} \mathrm{NMR}\left(\mathrm{CDCl}_{3}\right.$, $500 \mathrm{MHz}): \delta_{\mathrm{H}} 0.97$ (s, 3H, 18- $\left.\mathrm{H}_{3}\right), 1.05\left(\mathrm{~s}, 3 \mathrm{H}, 19-\mathrm{H}_{3}\right), 1.15$ $(\mathrm{m}, 2 \mathrm{H}), 1.47-1.76$ (overlapping $\mathrm{m}, 8 \mathrm{H}), 1.88(\mathrm{~m}, 2 \mathrm{H}), 1.94$ $(\mathrm{m}, 1 \mathrm{H}), 2.03\left(\mathrm{~s}, 3 \mathrm{H}, \mathrm{Ac}-\mathrm{CH}_{3}\right), 2.04(\mathrm{~m}, 1 \mathrm{H}), 2.35(\mathrm{~m}, 3 \mathrm{H})$, 3.24 (d, $1 \mathrm{H}, J=16.5 \mathrm{~Hz}$, one of $\left.4^{\prime}-\mathrm{H}\right), 3.62(\mathrm{~d}, 1 \mathrm{H}, J=16.5$ $\mathrm{Hz}$, the other $\left.4^{\prime}-\mathrm{H}\right), 3.83$ (s, 3H, 4'-OMe), 4.61 (m, 1H, 3$\mathrm{H}), 5.40(\mathrm{~m}, 1 \mathrm{H}, 6-\mathrm{H}), 6.90\left(\mathrm{~d}, 2 \mathrm{H}, J=8.6 \mathrm{~Hz}, 3^{\prime \prime}-\mathrm{H}\right.$ and $\left.5^{\prime \prime}-\mathrm{H}\right), 7.58\left(\mathrm{~d}, 2 \mathrm{H}, J=8.6 \mathrm{~Hz}, 2^{\prime \prime}-\mathrm{H}\right.$ and $\left.6^{\prime \prime}-\mathrm{H}\right) ;{ }^{13} \mathrm{C} \mathrm{NMR}$ $\left(\mathrm{CDCl}_{3}, 125 \mathrm{MHz}\right): \delta_{\mathrm{C}} 14.3(\mathrm{C}-18), 19.3(\mathrm{C}-19), 20.1\left(\mathrm{CH}_{2}\right)$, $21.4\left(\mathrm{Ac}-\mathrm{CH}_{3}\right), 27.6\left(\mathrm{CH}_{2}\right), 30.6\left(\mathrm{CH}_{2}\right), 31.0(\mathrm{CH}), 31.6$ $\left(\mathrm{CH}_{2}\right), 36.7(\mathrm{C}-10), 36.8\left(\mathrm{CH}_{2}\right), 37.5\left(\mathrm{CH}_{2}\right), 38.0\left(\mathrm{CH}_{2}\right)$, $44.2\left(\mathrm{CH}_{2}\right), 47.1(\mathrm{C}-13), 48.5(\mathrm{CH}), 49.9(\mathrm{CH}), 55.3\left(4^{\prime \prime}-\right.$ OMe), 73.6 (C-3), 88.4 (C-16), 114.1 (2C, C-3" and C-5"), $121.5\left(\mathrm{C}-1^{\prime \prime}\right), 121.7$ (C-6), 128.4 (2C, C-2" and C-6"), 139.8 (C-5), 154.9 (C-3'), 161.1 (C-4"), 170.4 (Ac-CO), 216.4 (C17); ESI-MS $515[\mathrm{M}+\mathrm{Na}]^{+}$; Anal. Calcd. for $\mathrm{C}_{30} \mathrm{H}_{37} \mathrm{NO}_{5} \mathrm{C}$ 73.29; H 7.59. Found C 73.42; H 7.74.

8c: $\mathrm{mp} 197-200{ }^{\circ} \mathrm{C}, R_{\mathrm{f}}=0.34$ (ss A); ${ }^{1} \mathrm{H} \mathrm{NMR}\left(\mathrm{CDCl}_{3}\right.$, $500 \mathrm{MHz}): \delta_{\mathrm{H}} 0.88(\mathrm{~m}, 1 \mathrm{H}), 1.07\left(\mathrm{~m}, 3 \mathrm{H}, 18-\mathrm{H}_{3}\right), 1.10(\mathrm{~s}$, $\left.3 \mathrm{H}, 19-\mathrm{H}_{3}\right), 1.15(\mathrm{~m}, 2 \mathrm{H}), 1.34(\mathrm{~m}, 1 \mathrm{H}), 1.52-1.65(\mathrm{~m}, 3 \mathrm{H})$, $1.70-1.81(\mathrm{~m}, 2 \mathrm{H}), 1.88-1.99(\mathrm{~m}, 3 \mathrm{H}), 2.03\left(\mathrm{~s}, 3 \mathrm{H}, \mathrm{Ac}^{-\mathrm{CH}_{3}}\right.$ ), $2.04(\mathrm{~m}, 1 \mathrm{H}), 2.09(\mathrm{~m}, 1 \mathrm{H}), 2.23(\mathrm{dd}, 1 \mathrm{H}, J=13.0 \mathrm{~Hz}, J$ $=5.6 \mathrm{~Hz}), 2.34(\mathrm{~m}, 2 \mathrm{H}), 3.11(\mathrm{~d}, 1 \mathrm{H}, J=16.3 \mathrm{~Hz}$, one of $\left.4^{\prime}-\mathrm{H}\right), 3.65\left(\mathrm{~d}, 1 \mathrm{H}, J=16.3 \mathrm{~Hz}\right.$, the other $\left.4^{\prime}-\mathrm{H}\right), 3.83(\mathrm{~s}, 3 \mathrm{H}$, 4"-OMe), $4.61(\mathrm{~m}, 1 \mathrm{H}, 3-\mathrm{H}), 5.39(\mathrm{~m}, 1 \mathrm{H}, 6-\mathrm{H}), 6.90(\mathrm{~d}$, $2 \mathrm{H}, J=8.4 \mathrm{~Hz}, 3^{\prime \prime}-\mathrm{H}$ and $\left.5^{\prime \prime}-\mathrm{H}\right), 7.58(\mathrm{~d}, 2 \mathrm{H}, J=8.4 \mathrm{~Hz}$, $2^{\prime \prime}-\mathrm{H}$ and $\left.6 "-\mathrm{H}\right) ;{ }^{13} \mathrm{C} \mathrm{NMR}\left(\mathrm{CDCl}_{3}, 125 \mathrm{MHz}\right): \delta_{\mathrm{C}} 13.0(\mathrm{C}-$ $18), 19.3$ (C-19), $20.1\left(\mathrm{CH}_{2}\right), 21.4\left(\mathrm{Ac}-\mathrm{CH}_{3}\right), 27.6\left(\mathrm{CH}_{2}\right)$, $29.7\left(\mathrm{CH}_{2}\right), 30.8\left(\mathrm{CH}_{2}\right), 30.9(\mathrm{CH}), 31.9\left(\mathrm{CH}_{2}\right), 36.8(\mathrm{C}-$ 10), $36.9\left(\mathrm{CH}_{2}\right), 38.0\left(\mathrm{CH}_{2}\right), 45.7\left(\mathrm{CH}_{2}\right), 46.5(\mathrm{CH}), 46.8$ (C-13), $50.2(\mathrm{CH}), 55.3$ (4"-OMe), 73.6 (C-3), 88.4 (C-16), 114.1 (2C, C-3" and C-5"), $121.4(\mathrm{C}-6), 121.5\left(\mathrm{C}-1^{\prime \prime}\right), 128.4$ (2C, C-2" and C-6"), $140.0(\mathrm{C}-5), 154.5\left(\mathrm{C}-3^{\prime}\right), 161.1\left(\mathrm{C}-4^{\prime \prime}\right)$, 170.5 (Ac-CO), 215.6 (C-17); ESI-MS 515 [M+Na] ${ }^{+}$; Anal. Calcd. for $\mathrm{C}_{30} \mathrm{H}_{37} \mathrm{NO}_{5} \mathrm{C}$ 73.29; H 7.59. Found C 73.39; H 7.78.

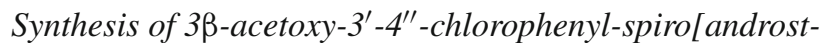
5-ene-16,5'-2'-isoxazolin]-17-one epimers ( $\mathbf{7 d}$ and $\mathbf{8 d})$

According to Sect. 4.1, $N$-hydroxy-4-chlorobenzenecarboximidoyl chloride (5d, $285 \mathrm{mg}$ ) was used. After purification with $\mathrm{CH}_{2} \mathrm{Cl}_{2}$ as eluent, $7 \mathbf{d}(357 \mathrm{mg}, 72 \%)$ and $\mathbf{8 d}(40$ $\mathrm{mg}, 8 \%$ ) were obtained as white solids (sequence of elution: 8d $>7 d$ ).

7d: mp 226-228 ${ }^{\circ} \mathrm{C}, R_{\mathrm{f}}=0.40\left(\right.$ ss A); ${ }^{1} \mathrm{H} \mathrm{NMR}\left(\mathrm{CDCl}_{3}\right.$, $500 \mathrm{MHz}): \delta_{\mathrm{H}} 0.96\left(\mathrm{~s}, 3 \mathrm{H}, 18-\mathrm{H}_{3}\right), 1.05\left(\mathrm{~s}, 3 \mathrm{H}, 19-\mathrm{H}_{3}\right), 1.14$ (m, 2H), 1.47-1.76 (overlapping m, 8H), $1.88(\mathrm{~m}, 2 \mathrm{H}), 1.95$ $(\mathrm{m}, 1 \mathrm{H}), 2.03\left(\mathrm{~s}, 3 \mathrm{H}, \mathrm{Ac}-\mathrm{CH}_{3}\right), 2.04(\mathrm{~m}, 1 \mathrm{H}), 2.36(\mathrm{~m}, 3 \mathrm{H})$, $3.24\left(\mathrm{~d}, 1 \mathrm{H}, J=16.6 \mathrm{~Hz}\right.$, one of $\left.4^{\prime}-\mathrm{H}\right), 3.62(\mathrm{~d}, 1 \mathrm{H}, J=16.6$ $\mathrm{Hz}$, the other $\left.4^{\prime}-\mathrm{H}\right), 4.61(\mathrm{~m}, 1 \mathrm{H}, 3-\mathrm{H}), 5.41(\mathrm{~m}, 1 \mathrm{H}, 6-\mathrm{H})$, $7.37\left(\mathrm{~d}, 2 \mathrm{H}, J=8.6 \mathrm{~Hz}, 3^{\prime \prime}-\mathrm{H}\right.$ and $\left.5^{\prime \prime}-\mathrm{H}\right), 7.58(\mathrm{~d}, 2 \mathrm{H}, J$ $=8.6 \mathrm{~Hz}, 2^{\prime \prime}-\mathrm{H}$ and $\left.6^{\prime \prime}-\mathrm{H}\right) ;{ }^{13} \mathrm{C} \mathrm{NMR}\left(\mathrm{CDCl}_{3}, 125 \mathrm{MHz}\right)$ : $\delta_{\mathrm{C}} 14.3(\mathrm{C}-18), 19.3(\mathrm{C}-19), 20.1\left(\mathrm{CH}_{2}\right), 21.4\left(\mathrm{Ac}-\mathrm{CH}_{3}\right)$, $27.6\left(\mathrm{CH}_{2}\right), 30.6\left(\mathrm{CH}_{2}\right), 31.0(\mathrm{CH}), 31.6\left(\mathrm{CH}_{2}\right), 36.7(\mathrm{C}-$ 10), $36.8\left(\mathrm{CH}_{2}\right), 37.5\left(\mathrm{CH}_{2}\right), 38.0\left(\mathrm{CH}_{2}\right), 43.7\left(\mathrm{CH}_{2}\right), 47.1$ (C-13), $48.5(\mathrm{CH}), 49.9(\mathrm{CH}), 73.6(\mathrm{C}-3), 88.9(\mathrm{C}-16), 121.6$ (C-6), $127.5\left(\mathrm{C}-1^{\prime \prime}\right), 128.0$ (2C, C-3" and C-5") 129.0 (2C, C-2" and C-6"), 136.2 (C-4"), 139.9 (C-5), $154.4\left(\mathrm{C}-3^{\prime}\right)$, 170.4 (Ac-CO), 216.0 (C-17); ESI-MS 519 [M+Na] ${ }^{+}$; Anal. Calcd. for $\mathrm{C}_{29} \mathrm{H}_{34} \mathrm{ClNO}_{4} \mathrm{C} 70.22 ; \mathrm{H}$ 6.91. Found C 70.37; H 7.08.

8d: Decomposed above $190{ }^{\circ} \mathrm{C}, R_{\mathrm{f}}=0.54(\mathrm{ss} \mathrm{A}) ;{ }^{1} \mathrm{HNMR}$ $\left(\mathrm{CDCl}_{3}, 500 \mathrm{MHz}\right): \delta_{\mathrm{H}} 1.06(\mathrm{~m}, 1 \mathrm{H}), 1.06\left(\mathrm{~s}, 3 \mathrm{H}, 19-\mathrm{H}_{3}\right)$, $1.10\left(\mathrm{~s}, 3 \mathrm{H}, 18-\mathrm{H}_{3}\right), 1.14(\mathrm{~m}, 2 \mathrm{H}), 1.33(\mathrm{~m}, 1 \mathrm{H}), 1.52-1.65$ (m, 3H), 1.70-1.79 (m, 2H), 1.87-1.97 (m, 3H), $2.03(\mathrm{~s}, 3 \mathrm{H}$, Ac- $\left.\mathrm{CH}_{3}\right), 2.05(\mathrm{~m}, 1 \mathrm{H}), 2.08(\mathrm{~m}, 1 \mathrm{H}), 2.23(\mathrm{dd}, 1 \mathrm{H}, J=12.9$ $\mathrm{Hz}, J=5.3 \mathrm{~Hz}), 2.34(\mathrm{~m}, 2 \mathrm{H}), 3.10(\mathrm{~d}, 1 \mathrm{H}, J=16.4 \mathrm{~Hz}$, one of $\left.4^{\prime}-\mathrm{H}\right), 3.64\left(\mathrm{~d}, 1 \mathrm{H}, J=16.4 \mathrm{~Hz}\right.$, the other $\left.4^{\prime}-\mathrm{H}\right), 4.60$ (m, 1H, 3-H), 5.39 (m, 1H, 6-H), 7.37 (d, 2H, $J=8.0 \mathrm{~Hz}$, $3^{\prime \prime}-\mathrm{H}$ and $\left.5^{\prime \prime}-\mathrm{H}\right), 7.57\left(\mathrm{~d}, 2 \mathrm{H}, J=8.0 \mathrm{~Hz}, 2^{\prime \prime}-\mathrm{H}\right.$ and $\left.6^{\prime \prime}-\mathrm{H}\right)$; ${ }^{13} \mathrm{C} \mathrm{NMR}\left(\mathrm{CDCl}_{3}, 125 \mathrm{MHz}\right): \delta_{\mathrm{C}} 13.0(\mathrm{C}-18), 19.3(\mathrm{C}-19)$, $20.1\left(\mathrm{CH}_{2}\right), 21.4\left(\mathrm{Ac}-\mathrm{CH}_{3}\right), 27.6\left(\mathrm{CH}_{2}\right), 30.8\left(\mathrm{CH}_{2}\right), 30.9$ $(\mathrm{CH}), 31.8\left(\mathrm{CH}_{2}\right), 36.8(\mathrm{C}-10), 36.9\left(\mathrm{CH}_{2}\right), 37.9\left(\mathrm{CH}_{2}\right), 38.0$ $\left(\mathrm{CH}_{2}\right), 45.2\left(\mathrm{CH}_{2}\right), 46.4(\mathrm{CH}), 46.8(\mathrm{C}-13), 50.2(\mathrm{CH}), 73.6$ (C-3), 88.9 (C-16), 121.4 (C-6), 127.5 (C-1"), 128.1 (2C, C$3^{\prime \prime}$ and C-5"), 129.0 (2C, C-2" and C-6" $), 136.2\left(\mathrm{C}-4^{\prime \prime}\right), 140.0$ (C-5), 154.1 (C-3'), 170.5 (Ac-CO), 215.2 (C-17); ESI-MS $519[\mathrm{M}+\mathrm{Na}]^{+}$; Anal. Calcd. for $\mathrm{C}_{29} \mathrm{H}_{34} \mathrm{ClNO}_{4} \mathrm{C} 70.22 ; \mathrm{H}$ 6.91. Found C 70.05; H 7.05.

Synthesis of $3 \beta$-acetoxy- $3^{\prime}-4^{\prime \prime}$-nitrophenylspiro[androst-5-ene-16,5'-2'-isoxazolin]-17-one (7e)

According to Sect. 4.1, $N$-hydroxy-4-nitrobenzenecarboximidoyl chloride (5e, $300 \mathrm{mg}$ ) was used. After purification with EtOAc/ $\mathrm{CH}_{2} \mathrm{Cl}_{2}=1: 99$ as eluent, 7 e $(360 \mathrm{mg}, 71$ $\%)$ was obtained as a pale-yellow solid.

7e: $\mathrm{mp} 233-235^{\circ} \mathrm{C}, R_{\mathrm{f}}=38(\mathrm{ss} \mathrm{A}) ;{ }^{1} \mathrm{H} \mathrm{NMR}\left(\mathrm{CDCl}_{3}, 500\right.$ MHz): $\delta_{\mathrm{H}} 0.97\left(\mathrm{~s}, 3 \mathrm{H}, 18-\mathrm{H}_{3}\right), 1.06\left(\mathrm{~s}, 3 \mathrm{H}, 19-\mathrm{H}_{3}\right), 1.15(\mathrm{~m}$, 
2H), 1.48-1.79 (overlapping m, 8H), $1.88(\mathrm{~m}, 2 \mathrm{H}), 1.96$ (m, 1H), 2.03 (s, 3H, Ac-CH $), 2.04(\mathrm{~m}, 1 \mathrm{H}), 2.35(\mathrm{~m}, 3 \mathrm{H}), 3.30$ $\left(\mathrm{d}, 1 \mathrm{H}, J=16.6 \mathrm{~Hz}\right.$, one of $\left.4^{\prime}-\mathrm{H}\right), 3.65(\mathrm{~d}, 1 \mathrm{H}, J=16.6 \mathrm{~Hz}$, the other $\left.4^{\prime}-\mathrm{H}\right), 4.61(\mathrm{~m}, 1 \mathrm{H}, 3-\mathrm{H}), 5.40(\mathrm{~m}, 1 \mathrm{H}, 6-\mathrm{H}), 7.80(\mathrm{~d}$, $2 \mathrm{H}, J=8.6 \mathrm{~Hz}, 2^{\prime \prime}-\mathrm{H}$ and $\left.6^{\prime \prime}-\mathrm{H}\right), 8.25(\mathrm{~d}, 2 \mathrm{H}, J=8.6 \mathrm{~Hz}$, $3^{\prime \prime}-\mathrm{H}$ and $\left.5^{\prime \prime}-\mathrm{H}\right) ;{ }^{13} \mathrm{C} \mathrm{NMR}\left(\mathrm{CDCl}_{3}, 125 \mathrm{MHz}\right): \delta_{\mathrm{C}} 14.4(\mathrm{C}-$ 18), 19.3 (C-19), $20.1\left(\mathrm{CH}_{2}\right), 21.4\left(\mathrm{Ac}-\mathrm{CH}_{3}\right), 27.6\left(\mathrm{CH}_{2}\right)$, $30.6\left(\mathrm{CH}_{2}\right), 31.0(\mathrm{CH}), 31.6\left(\mathrm{CH}_{2}\right), 36.7(\mathrm{C}-10), 36.8\left(\mathrm{CH}_{2}\right)$, $37.4\left(\mathrm{CH}_{2}\right), 38.0\left(\mathrm{CH}_{2}\right), 43.1\left(\mathrm{CH}_{2}\right), 47.1(\mathrm{C}-13), 48.6(\mathrm{CH})$, $49.9(\mathrm{CH}), 73.6$ (C-3), 89.7 (C-16), 121.5 (C-6), 124.0 (2C, $\mathrm{C}-3^{\prime \prime}$ and $\left.\mathrm{C}-5^{\prime \prime}\right), 127.5$ (2C, C-2" and $\left.\mathrm{C}-6^{\prime \prime}\right), 135.1$ (C$1^{\prime \prime}$ ), 140.0 (C-5), 148.5 (C-4"), 153.8 (C-3'), 170.4 (AcCO), 215.5 (C-17); ESI-MS $530[\mathrm{M}+\mathrm{Na}]^{+}$; Anal. Calcd. for $\mathrm{C}_{29} \mathrm{H}_{34} \mathrm{~N}_{2} \mathrm{O}_{6} \mathrm{C}$ 68.76; H 6.76. Found C 68.89; H 6.92.

General procedure for the deacetylation of 16-spiroisoxazolyl derivatives (7a-e)

Compound 7a-e $(0.60 \mathrm{mmol})$ was dissolved in $\mathrm{MeOH}(10$ $\mathrm{mL}$ ), and $\mathrm{KOH}(50 \mathrm{mg}, 0.89 \mathrm{mmol})$ was added. The solution was stirred at room temperature for $8 \mathrm{~h}$, then diluted with water and neutralized with dilute $\mathrm{HCl}$. The resulting precipitate was filtered, washed with water, and dried.

Synthesis of 3 $\beta$-hydroxy-3'-phenyl-spiro[androst-5-ene$16,5^{\prime} S$-2'-isoxazolin]-17-one (11a)

Substrate: 7a $(277 \mathrm{mg})$. 11a was obtained as a white solid (229 mg, 91\%), mp 223-226 ${ }^{\circ} \mathrm{C}, R_{\mathrm{f}}=0.37$ (ss B); ${ }^{1} \mathrm{H}$ NMR $\left(\mathrm{CDCl}_{3}, 500 \mathrm{MHz}\right): \delta_{\mathrm{H}} 0.97$ (s, 3H, 18- $\left.\mathrm{H}_{3}\right), 1.04$ (s, 3H, 19$\left.\mathrm{H}_{3}\right), 1.11(\mathrm{~m}, 2 \mathrm{H}), 1.52(\mathrm{~m}, 3 \mathrm{H}), 1.65-1.76$ (overlapping $\mathrm{m}$, $5 \mathrm{H}), 1.86(\mathrm{~m}, 2 \mathrm{H}), 1.95(\mathrm{~m}, 1 \mathrm{H}), 2.05(\mathrm{~m}, 1 \mathrm{H}), 2.24(\mathrm{~m}, 1 \mathrm{H})$, $2.32(\mathrm{~m}, 1 \mathrm{H}), 2.39(\mathrm{~m}, 1 \mathrm{H}), 3.28(\mathrm{~d}, 1 \mathrm{H}, J=16.6 \mathrm{~Hz}$, one of $\left.4^{\prime}-\mathrm{H}\right), 3.54(\mathrm{~m}, 1 \mathrm{H}, 3-\mathrm{H}), 3.65(\mathrm{~d}, 1 \mathrm{H}, J=16.6 \mathrm{~Hz}$, the other $\left.4^{\prime}-\mathrm{H}\right), 5.37$ (m, 1H, 6-H), $7.40\left(\mathrm{~m}, 3 \mathrm{H}, 3^{\prime \prime}-\mathrm{H}, 4^{\prime \prime}-\mathrm{H}\right.$ and $\left.5^{\prime \prime}-\mathrm{H}\right), 7.64$ (m, 2H, 2"- $\mathrm{H}$ and $\left.66^{\prime \prime}-\mathrm{H}\right),{ }^{13} \mathrm{C} \mathrm{NMR}\left(\mathrm{CDCl}_{3}, 125\right.$ MHz): $\left.\delta_{\mathrm{C}} 14.3(\mathrm{C}-18), 19.4(\mathrm{C}-19), 20.2\left(\mathrm{CH}_{2}\right), 30.7 \mathrm{CH}_{2}\right)$, $31.0(\mathrm{CH}), 31.5\left(\mathrm{CH}_{2}\right), 31.6\left(\mathrm{CH}_{2}\right), 36.6(\mathrm{C}-10), 37.0\left(\mathrm{CH}_{2}\right)$, $37.5\left(\mathrm{CH}_{2}\right), 42.1\left(\mathrm{CH}_{2}\right), 44.0\left(\mathrm{CH}_{2}\right), 47.1(\mathrm{C}-13), 48.6(\mathrm{CH})$, $50.0(\mathrm{CH}), 71.5(\mathrm{C}-3), 88.7$ (C-16), $120.7(\mathrm{C}-6), 126.8(2 \mathrm{C}$, $\mathrm{C}-3^{\prime \prime}$ and $\left.\mathrm{C}-5^{\prime \prime}\right), 128.7$ (2C, C-2" and C-6"), $129.0\left(\mathrm{C}-1^{\prime \prime}\right)$, $130.2\left(\mathrm{C}-4^{\prime \prime}\right), 141.0$ (C-5), $155.4\left(\mathrm{C}-3^{\prime}\right), 216.3$ (C-17); ESIMS $443[\mathrm{M}+\mathrm{Na}]^{+}$; Anal. Calcd. for $\mathrm{C}_{27} \mathrm{H}_{33} \mathrm{NO}_{3} \mathrm{C} 77.29 ; \mathrm{H}$ 7.93. Found C 77.43; H 8.15.

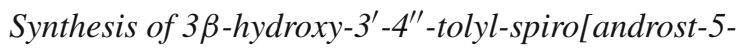
ene-16,5'S-2'-isoxazolin]-17-one (11b)

Substrate: $\mathbf{7 b}(285 \mathrm{mg})$. 11b was obtained as a white solid (239 mg, $92 \%$ ), mp $270-272{ }^{\circ} \mathrm{C}, R_{\mathrm{f}}=0.37$ (ss B); ${ }^{1} \mathrm{H}$ NMR $\left(\mathrm{CDCl}_{3}, 500 \mathrm{MHz}\right): \delta_{\mathrm{H}} 0.96\left(\mathrm{~s}, 3 \mathrm{H}, 18-\mathrm{H}_{3}\right), 1.04(\mathrm{~s}, 3 \mathrm{H}$, 19- $\left.\mathrm{H}_{3}\right), 1.10(\mathrm{~m}, 2 \mathrm{H}), 1.51(\mathrm{~m}, 3 \mathrm{H}), 1.65-1.77$ (overlapping $\mathrm{m}, 5 \mathrm{H}), 1.86(\mathrm{~m}, 2 \mathrm{H}), 1.94(\mathrm{~m}, 1 \mathrm{H}), 2.03(\mathrm{~m}, 1 \mathrm{H}), 2.24(\mathrm{~m}$,
$1 \mathrm{H}), 2.36(\mathrm{~m}, 2 \mathrm{H}), 2.37\left(\mathrm{~s}, 3 \mathrm{H}, 4^{\prime \prime}-\mathrm{CH}_{3}\right), 3.26(\mathrm{~d}, 1 \mathrm{H}, J$ $=16.5 \mathrm{~Hz}$, one of $\left.4^{\prime}-\mathrm{H}\right), 3.54(\mathrm{~m}, 1 \mathrm{H}, 3-\mathrm{H}), 3.63(\mathrm{~d}, 1 \mathrm{H}$, $J=16.5 \mathrm{~Hz}$, the other $\left.4^{\prime}-\mathrm{H}\right), 5.37(\mathrm{~m}, 1 \mathrm{H}, 6-\mathrm{H}), 7.19(\mathrm{~d}$, $2 \mathrm{H}, J=8.0 \mathrm{~Hz}, 3^{\prime \prime}-\mathrm{H}$ and $\left.5^{\prime \prime}-\mathrm{H}\right), 7.53(\mathrm{~d}, 2 \mathrm{H}, J=8.0 \mathrm{~Hz}$, $2^{\prime \prime}-\mathrm{H}$ and $\left.6{ }^{\prime \prime}-\mathrm{H}\right) ;{ }^{13} \mathrm{C} \mathrm{NMR}\left(\mathrm{CDCl}_{3}, 125 \mathrm{MHz}\right): \delta_{\mathrm{C}}=14.3$ (C-18), $19.4(\mathrm{C}-19), 20.2\left(\mathrm{CH}_{2}\right), 21.4\left(4^{\prime \prime}-\mathrm{CH}_{3}\right), 30.7\left(\mathrm{CH}_{2}\right)$, $31.0(\mathrm{CH}), 31.5\left(\mathrm{CH}_{2}\right), 31.6\left(\mathrm{CH}_{2}\right), 36.6(\mathrm{C}-10), 37.1\left(\mathrm{CH}_{2}\right)$, $37.6\left(\mathrm{CH}_{2}\right), 42.1\left(\mathrm{CH}_{2}\right), 44.2\left(\mathrm{CH}_{2}\right), 47.1(\mathrm{C}-13), 48.6(\mathrm{CH})$, $50.0(\mathrm{CH}), 71.5(\mathrm{C}-3), 88.5(\mathrm{C}-16), 120.8(\mathrm{C}-6), 126.1(\mathrm{C}-$ $\left.1^{\prime \prime}\right), 126.8\left(2 \mathrm{C}, \mathrm{C}-2^{\prime \prime}\right.$ and $\left.\mathrm{C}-6^{\prime \prime}\right), 129.4\left(2 \mathrm{C}, \mathrm{C}-3^{\prime \prime}\right.$ and $\left.\mathrm{C}-5^{\prime \prime}\right)$, $140.5\left(\mathrm{C}-4^{\prime \prime}\right), 140.9$ (C-5), 155.4 (C-3'), 216.4 (C-17); ESIMS $435[\mathrm{M}+\mathrm{H}]^{+}$; Anal. Calcd. for $\mathrm{C}_{28} \mathrm{H}_{35} \mathrm{NO}_{3} \mathrm{C} 77.56 ; \mathrm{H}$ 8.14. Found C 77.42; H 8.20.

Synthesis of $3 \beta$-hydroxy-3'-4" -methoxyphenylspiro[androst-5-ene-16,5'S-2'-isoxazolin]-17-one (11c)

Substrate: 7c $(295 \mathrm{mg})$. 11c was obtained as a white solid (245 mg, $91 \%$ ), mp $254-255{ }^{\circ} \mathrm{C}, R_{\mathrm{f}}=0.29$ (ss B); ${ }^{1} \mathrm{H}$ NMR $\left(\mathrm{CDCl}_{3}, 500 \mathrm{MHz}\right): \delta_{\mathrm{H}} 0.96\left(\mathrm{~s}, 3 \mathrm{H}, 18-\mathrm{H}_{3}\right), 1.04$ (s, 3H, 19$\left.\mathrm{H}_{3}\right), 1.10(\mathrm{~m}, 2 \mathrm{H}), 1.52(\mathrm{~m}, 3 \mathrm{H}), 1.65-1.72$ (overlapping $\mathrm{m}$, $5 \mathrm{H}), 1.86(\mathrm{~m}, 2 \mathrm{H}), 1.94(\mathrm{~m}, 1 \mathrm{H}), 2.03(\mathrm{~m}, 1 \mathrm{H}), 2.24(\mathrm{~m}, 1 \mathrm{H})$, $2.32(\mathrm{~m}, 1 \mathrm{H}), 2.38(\mathrm{~m}, 1 \mathrm{H}), 3.25(\mathrm{~d}, 1 \mathrm{H}, J=16.5 \mathrm{~Hz}$, one of $\left.4^{\prime}-\mathrm{H}\right), 3.54(\mathrm{~m}, 1 \mathrm{H}, 3-\mathrm{H}), 3.62(\mathrm{~d}, 1 \mathrm{H}, J=16.5 \mathrm{~Hz}$, the other $\left.4^{\prime}-\mathrm{H}\right), 3.83\left(\mathrm{~s}, 3 \mathrm{H}, 4^{\prime \prime}-\mathrm{OMe}\right), 5.37(\mathrm{~m}, 1 \mathrm{H}, 6-\mathrm{H}), 6.90$ $\left(\mathrm{d}, 2 \mathrm{H}, J=8.5 \mathrm{~Hz}, 3^{\prime \prime}-\mathrm{H}\right.$ and $\left.5^{\prime \prime}-\mathrm{H}\right), 7.58(\mathrm{~d}, 2 \mathrm{H}, J=8.5 \mathrm{~Hz}$, $2^{\prime \prime}-\mathrm{H}$ and $\left.6^{\prime \prime}-\mathrm{H}\right) ;{ }^{13} \mathrm{C} \mathrm{NMR}\left(\mathrm{CDCl}_{3}, 125 \mathrm{MHz}\right): \delta_{\mathrm{C}} 14.3(\mathrm{C}-$ 18), 19.4 (C-19), $20.2\left(\mathrm{CH}_{2}\right), 30.7\left(\mathrm{CH}_{2}\right), 31.0(\mathrm{CH}), 31.5$ $\left(\mathrm{CH}_{2}\right), 31.6\left(\mathrm{CH}_{2}\right), 36.6(\mathrm{C}-10), 37.0\left(\mathrm{CH}_{2}\right), 37.6\left(\mathrm{CH}_{2}\right)$, $42.1\left(\mathrm{CH}_{2}\right), 44.3\left(\mathrm{CH}_{2}\right), 47.1(\mathrm{C}-13), 48.6(\mathrm{CH}), 50.0(\mathrm{CH})$, 55.3 (4"-OMe), $71.5(\mathrm{C}-3), 88.4(\mathrm{C}-16), 114.1$ (2C, C-3" and C-5"), 120.8 (C-6), 121.5 (C-1"), 128.4 (2C, C-2" and C-6"), 140.9 (C-5), $155.0\left(\mathrm{C}-3^{\prime}\right), 161.1\left(\mathrm{C}-4^{\prime \prime}\right), 216.5$ (C-17); ESIMS $473[\mathrm{M}+\mathrm{Na}]^{+}$; Anal. Calcd. for $\mathrm{C}_{28} \mathrm{H}_{35} \mathrm{NO}_{4} \mathrm{C} 74.80 ; \mathrm{H}$ 7.85. Found C 74.96; H 8.02.

Synthesis of 3 $\beta$-hydroxy-3'-4" -chlorophenylspiro [androst-5-ene-16,5'S-2'-isoxazolin]-17-one (11d)

Substrate: 7d (298 mg). 11d was obtained as a white solid $(259 \mathrm{mg}, 95 \%), \mathrm{mp} 256-259{ }^{\circ} \mathrm{C}, R_{\mathrm{f}}=0.38$ (ss B); ${ }^{1} \mathrm{H} \mathrm{NMR}$ $\left(\mathrm{CDCl}_{3}, 500 \mathrm{MHz}\right): \delta_{\mathrm{H}} 0.95\left(\mathrm{~s}, 3 \mathrm{H}, 18-\mathrm{H}_{3}\right), 1.04(\mathrm{~s}, 3 \mathrm{H}$, 19- $\left.\mathrm{H}_{3}\right), 1.12(\mathrm{~m}, 2 \mathrm{H}), 1.47-1.78$ (overlapping $\left.\mathrm{m}, 8 \mathrm{H}\right), 1.87$ $(\mathrm{m}, 2 \mathrm{H}), 1.96(\mathrm{~m}, 1 \mathrm{H}), 2.05(\mathrm{~m}, 1 \mathrm{H}), 2.24(\mathrm{~m}, 1 \mathrm{H}), 2.34(\mathrm{~m}$, $1 \mathrm{H}), 2.41(\mathrm{~m}, 1 \mathrm{H}), 3.25\left(\mathrm{~d}, 1 \mathrm{H}, J=16.6 \mathrm{~Hz}\right.$, one of $\left.4^{\prime}-\mathrm{H}\right)$, $3.54(\mathrm{~m}, 1 \mathrm{H}, 3-\mathrm{H}), 3.61\left(\mathrm{~d}, 1 \mathrm{H}, J=16.6 \mathrm{~Hz}\right.$, the other $\left.4^{\prime}-\mathrm{H}\right)$, $5.36(\mathrm{~m}, 1 \mathrm{H}, 6-\mathrm{H}), 7.36\left(\mathrm{~d}, 2 \mathrm{H}, J=8.6 \mathrm{~Hz}, 3^{\prime \prime}-\mathrm{H}\right.$ and $\left.5^{\prime \prime}-\mathrm{H}\right)$, $7.57\left(\mathrm{~d}, 2 \mathrm{H}, J=8.6 \mathrm{~Hz}, 2^{\prime \prime}-\mathrm{H}\right.$ and $\left.6^{\prime \prime}-\mathrm{H}\right) ;{ }^{13} \mathrm{C}-\mathrm{NMR}\left(\mathrm{CDCl}_{3}\right.$, $125 \mathrm{MHz}): \delta_{\mathrm{C}}=14.3(\mathrm{C}-18), 19.4(\mathrm{C}-19), 20.2\left(\mathrm{CH}_{2}\right), 30.6$ $\left(\mathrm{CH}_{2}\right), 31.0(\mathrm{CH}), 31.5\left(\mathrm{CH}_{2}\right), 31.6\left(\mathrm{CH}_{2}\right), 36.6(\mathrm{C}-10), 37.0$ $\left(\mathrm{CH}_{2}\right), 37.5\left(\mathrm{CH}_{2}\right), 42.1\left(\mathrm{CH}_{2}\right), 43.8\left(\mathrm{CH}_{2}\right), 47.1(\mathrm{C}-13)$, $48.6(\mathrm{CH}), 50.0(\mathrm{CH}), 71.4(\mathrm{C}-3), 89.0(\mathrm{C}-16), 120.7(\mathrm{C}-6)$, $127.5\left(\mathrm{C}-1^{\prime \prime}\right), 128.0$ (2C, C-3" and C-5"), 129.0 (2C, C-2" 
and C-6") $136.2\left(\mathrm{C}-4^{\prime \prime}\right), 141.0(\mathrm{C}-5), 154.5\left(\mathrm{C}-3^{\prime}\right), 216.1(\mathrm{C}-$ 17); ESI-MS $477[\mathrm{M}+\mathrm{H}]^{+}$; Anal. Calcd. for $\mathrm{C}_{27} \mathrm{H}_{32} \mathrm{ClNO}_{3}$ C 71.43; H 7.10. Found C 71.52; H 7.29.

Synthesis of 3 $\beta$-hydroxy-3'-4"-nitrophenylspiro[androst-5-ene-16,5'S-2'-isoxazolin]-17-one (11e)

Substrate: 7e (304 mg). 11e was obtained as a yellow solid (262 mg, $94 \%$ ), mp $265-268{ }^{\circ} \mathrm{C}, R_{\mathrm{f}}=0.37$ (ss B); ${ }^{1} \mathrm{H}$ NMR $\left(\mathrm{CDCl}_{3}, 500 \mathrm{MHz}\right): \delta_{\mathrm{H}} 0.98\left(\mathrm{~s}, 3 \mathrm{H}, 18-\mathrm{H}_{3}\right), 1.05$ (s, 3H, 19$\mathrm{H}_{3}$ ), 1.12 (m, 2H), 1.48-1.79 (overlapping $\left.\mathrm{m}, 8 \mathrm{H}\right), 1.87$ (m, $2 \mathrm{H}), 1.97(\mathrm{~m}, 1 \mathrm{H}), 2.05(\mathrm{~m}, 1 \mathrm{H}), 2.24(\mathrm{~m}, 1 \mathrm{H}), 2.33(\mathrm{~m}, 1 \mathrm{H})$, $2.42(\mathrm{~m}, 1 \mathrm{H}), 3.30\left(\mathrm{~d}, 1 \mathrm{H}, J=16.6 \mathrm{~Hz}\right.$, one of $\left.4^{\prime}-\mathrm{H}\right), 3.54$ $(\mathrm{m}, 1 \mathrm{H}, 3-\mathrm{H}), 3.66\left(\mathrm{~d}, 1 \mathrm{H}, J=16.6 \mathrm{~Hz}\right.$, the other $\left.4^{\prime}-\mathrm{H}\right), 5.40$ (m, 1H, 6-H), $7.81\left(\mathrm{~d}, 2 \mathrm{H}, J=8.7 \mathrm{~Hz}, 2^{\prime \prime}-\mathrm{H}\right.$ és $\left.6^{\prime \prime}-\mathrm{H}\right), 8.25$ (d, $2 \mathrm{H}, J=8.6 \mathrm{~Hz}, 3^{\prime \prime}-\mathrm{H}$ és $\left.5^{\prime \prime}-\mathrm{H}\right) ;{ }^{13} \mathrm{C} \mathrm{NMR}\left(\mathrm{CDCl}_{3}, 125\right.$ $\mathrm{MHz}): \delta_{\mathrm{C}} 14.0(\mathrm{C}-18), 19.0(\mathrm{C}-19), 19.8\left(\mathrm{CH}_{2}\right), 30.3\left(\mathrm{CH}_{2}\right)$, $30.6(\mathrm{CH}), 31.1\left(\mathrm{CH}_{2}\right), 31.2\left(\mathrm{CH}_{2}\right), 36.3(\mathrm{C}-10), 36.7\left(\mathrm{CH}_{2}\right)$, $37.0\left(\mathrm{CH}_{2}\right), 41.7\left(\mathrm{CH}_{2}\right), 42.8\left(\mathrm{CH}_{2}\right), 46.8(\mathrm{C}-13), 48.2(\mathrm{CH})$, $49.6(\mathrm{CH}), 71.1(\mathrm{C}-3), 89.4(\mathrm{C}-16), 120.2$ (C-6), 123.6 (2C, $\mathrm{C}-3^{\prime \prime}$ and $\left.\mathrm{C}-5^{\prime \prime}\right), 127.2\left(2 \mathrm{C}, \mathrm{C}-2^{\prime \prime}\right.$ and $\left.\mathrm{C}-6^{\prime \prime}\right), 134.7\left(\mathrm{C}-1^{\prime \prime}\right)$, 140.6 (C-5), 148.1 (C-4"), 153.5 (C-3'), 215.2 (C-17); ESIMS $488[\mathrm{M}+\mathrm{Na}]^{+}$; Anal. Calcd. for $\mathrm{C}_{27} \mathrm{H}_{32} \mathrm{~N}_{2} \mathrm{O}_{5} \mathrm{C} 69.81$; H 6.94. Found C 69.96; H 7.08.

General procedure for the reduction of 16-spiroisoxazolyl derivatives (11a-e)

Compound 11a-e $(0.45 \mathrm{mmol})$ was dissolved in a mixture of $\mathrm{MeOH}(10 \mathrm{~mL})$ and THF $(10 \mathrm{~mL})$, and $\mathrm{KBH}_{4}(50 \mathrm{mg}$, $0.93 \mathrm{mmol}$ ) was added. The solution was stirred at room temperature for $1 \mathrm{~h}$, then diluted with water and neutralized with dilute $\mathrm{HCl}$. The resulting precipitate was filtered, washed with water, and purified by flash chromatography with $\mathrm{EtOAc} / \mathrm{CH}_{2} \mathrm{Cl}_{2}=20: 80$ as eluent.

Synthesis of 3 $\beta$-hydroxy-3'-phenyl-spiro[androst-5-ene16,5'S-2'-isoxazolin]-17 $\beta$-ol (12a)

Substrate: 11a (189 mg). 12a was obtained as a white solid (186 mg, $98 \%$ ), mp 233-236 ${ }^{\circ} \mathrm{C}, R_{\mathrm{f}}=0.44$ (ss C); ${ }^{1} \mathrm{H}$ NMR (DMSO-d $6,500 \mathrm{MHz}): \delta_{\mathrm{H}} 0.67\left(\mathrm{~s}, 3 \mathrm{H}, 18-\mathrm{H}_{3}\right), 0.95(\mathrm{~s}, 3 \mathrm{H}$, 19- $\left.\mathrm{H}_{3}\right), 0.99(\mathrm{~m}, 2 \mathrm{H}), 1.18(\mathrm{~m}, 2 \mathrm{H}), 1.32-1.42$ (overlapping $\mathrm{m}, 3 \mathrm{H}), 1.48-1.56$ (overlapping $\mathrm{m}, 3 \mathrm{H}), 1.69(\mathrm{~m}, 1 \mathrm{H}), 1.76$ $(\mathrm{m}, 2 \mathrm{H}), 1.88(\mathrm{~m}, 1 \mathrm{H}), 2.00(\mathrm{~m}, 1 \mathrm{H}), 2.06-2.17$ (overlapping $\mathrm{m}, 2 \mathrm{H}), 3.00\left(\mathrm{~d}, 1 \mathrm{H}, J=17.1 \mathrm{~Hz}\right.$, one of $\left.4^{\prime}-\mathrm{H}\right), 3.27(\mathrm{~m}, 1 \mathrm{H}$, $3-\mathrm{H}), 3.62(\mathrm{~d}, 1 \mathrm{H}, J=4.9 \mathrm{~Hz}, 17-\mathrm{H}), 3.78(\mathrm{~d}, 1 \mathrm{H}, J=17.1$ $\mathrm{Hz}$, the other $\left.4^{\prime}-\mathrm{H}\right), 4.63(\mathrm{~d}, 1 \mathrm{H}, J=4.3 \mathrm{~Hz}, 3-\mathrm{OH}), 5.26$ $(\mathrm{m}, 1 \mathrm{H}, 6-\mathrm{H}), 5.33(\mathrm{~d}, 1 \mathrm{H}, J=4.9 \mathrm{~Hz}, 17-\mathrm{OH}), 7.42(\mathrm{~m}$, $3 \mathrm{H}, 3^{\prime \prime}-\mathrm{H}, 4^{\prime \prime}-\mathrm{H}$ and $\left.5^{\prime \prime}-\mathrm{H}\right), 7.66\left(\mathrm{~m}, 2 \mathrm{H}, 2^{\prime \prime}-\mathrm{H}\right.$ and $\left.6^{\prime \prime}-\mathrm{H}\right)$; ${ }^{13} \mathrm{C}$ NMR (DMSO-d $6,125 \mathrm{MHz}$ ): $\delta_{\mathrm{C}} 11.4$ (C-18), 19.1 (C19), $20.0\left(\mathrm{CH}_{2}\right), 30.8(\mathrm{CH}), 30.9\left(\mathrm{CH}_{2}\right), 31.3\left(\mathrm{CH}_{2}\right), 36.2$ $\left(\mathrm{CH}_{2}\right), 36.3(\mathrm{C}-10), 36.8\left(\mathrm{CH}_{2}\right), 41.1\left(\mathrm{CH}_{2}\right), 41.6(\mathrm{C}-13)$,
$42.1\left(\mathrm{CH}_{2}\right), 42.6\left(\mathrm{CH}_{2}\right), 47.7(\mathrm{CH}), 49.7(\mathrm{CH}), 69.9(\mathrm{C}-3)$, 85.1 (C-17), 94.9 (C-16), 120.7 (C-6), 126.2 (2C, C-3" and C-5") 128.7 (2C, C-2" and C-6"), $129.6\left(\mathrm{C}-4^{\prime \prime}\right), 129.9$ (C$\left.1^{\prime \prime}\right), 141.3(\mathrm{C}-5), 156.4\left(\mathrm{C}-3^{\prime}\right)$; ESI-MS 445 [M+Na] ${ }^{+}$; Anal. Calcd. for $\mathrm{C}_{27} \mathrm{H}_{35} \mathrm{NO}_{3} \mathrm{C}$ 76.92; $\mathrm{H}$ 8.37. Found $\mathrm{C} 77.06 ; \mathrm{H}$ 8.55 .

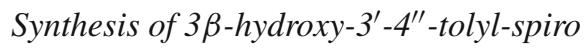
[androst-5-ene-16,5'S-2'-isoxazolin]-17 $\beta$-ol (12b)

Substrate: 11b (195 mg). 12b was obtained as a white solid $(186 \mathrm{mg}, 95 \%)$, mp 227-230 ${ }^{\circ} \mathrm{C}, R_{\mathrm{f}}=0.44(\mathrm{ss} \mathrm{C}) ;{ }^{1} \mathrm{H}$ NMR (DMSO-d $6,500 \mathrm{MHz}): \delta_{\mathrm{H}} 0.66\left(\mathrm{~s}, 3 \mathrm{H}, 18-\mathrm{H}_{3}\right), 0.95(\mathrm{~s}, 3 \mathrm{H}$, 19- $\left.\mathrm{H}_{3}\right), 0.99(\mathrm{~m}, 2 \mathrm{H}), 1.17(\mathrm{~m}, 2 \mathrm{H}), 1.32-1.40$ (overlapping $\mathrm{m}, 3 \mathrm{H}), 1.47-1.55$ (overlapping $\mathrm{m}, 3 \mathrm{H}), 1.67(\mathrm{~m}, 1 \mathrm{H}), 1.76$ $(\mathrm{m}, 2 \mathrm{H}), 1.89(\mathrm{~m}, 1 \mathrm{H}), 1.98(\mathrm{~m}, 1 \mathrm{H}), 2.07-2.16$ (overlapping $\mathrm{m}, 2 \mathrm{H}), 2.33\left(\mathrm{~s}, 3 \mathrm{H}, 4^{\prime \prime}-\mathrm{CH}_{3}\right), 2.96(\mathrm{~d}, 1 \mathrm{H}, J=17.1 \mathrm{~Hz}$, one of $\left.4^{\prime}-\mathrm{H}\right), 3.27(\mathrm{~m}, 1 \mathrm{H}, 3-\mathrm{H}), 3.61(\mathrm{~d}, 1 \mathrm{H}, J=4.8 \mathrm{~Hz}, 17-\mathrm{H})$, $3.74\left(\mathrm{~d}, 1 \mathrm{H}, J=17.1 \mathrm{~Hz}\right.$, the other $\left.4^{\prime}-\mathrm{H}\right), 4.60(\mathrm{~d}, 1 \mathrm{H}, J=4.4$ $\mathrm{Hz}, 3-\mathrm{OH}), 5.26(\mathrm{~m}, 1 \mathrm{H}, 6-\mathrm{H}), 5.30(\mathrm{~d}, 1 \mathrm{H}, J=4.9 \mathrm{~Hz}, 17-$ $\mathrm{OH}), 7.23\left(\mathrm{~d}, 2 \mathrm{H}, J=7.8 \mathrm{~Hz}, 3^{\prime \prime}-\mathrm{H}\right.$ and $\left.5^{\prime \prime}-\mathrm{H}\right), 7.55(\mathrm{~d}, 2 \mathrm{H}, J$ $=7.8 \mathrm{~Hz}, 2^{\prime \prime}-\mathrm{H}$ and $\left.6^{\prime \prime}-\mathrm{H}\right) ;{ }^{13} \mathrm{C} \mathrm{NMR}\left(\mathrm{DMSO}-\mathrm{d}_{6}, 125 \mathrm{MHz}\right)$ : $\delta_{C} 11.4(\mathrm{C}-18), 19.1(\mathrm{C}-19), 19.9\left(\mathrm{CH}_{2}\right), 20.9\left(4^{\prime \prime}-\mathrm{CH}_{3}\right), 30.7$ $(\mathrm{CH}), 30.9\left(\mathrm{CH}_{2}\right), 31.3\left(\mathrm{CH}_{2}\right), 36.1\left(\mathrm{CH}_{2}\right), 36.3(\mathrm{C}-10), 36.7$ $\left(\mathrm{CH}_{2}\right), 41.2\left(\mathrm{CH}_{2}\right), 41.6(\mathrm{C}-13), 42.1\left(\mathrm{CH}_{2}\right), 42.6\left(\mathrm{CH}_{2}\right)$, $47.7(\mathrm{CH}), 49.6(\mathrm{CH}), 69.9(\mathrm{C}-3), 85.0(\mathrm{C}-17), 94.6(\mathrm{C}-16)$, $120.1(\mathrm{C}-6), 126.2\left(2 \mathrm{C}, \mathrm{C}-2^{\prime \prime}\right.$ and C-6"), $127.1\left(\mathrm{C}-1^{\prime \prime}\right), 129.2$ (2C, C-3" and C-5"), 139.2 (C-4"), 141.2 (C-5), 156.2 (C$\left.3^{\prime}\right)$; ESI-MS $437[\mathrm{M}+\mathrm{H}]^{+}$; Anal. Calcd. for $\mathrm{C}_{28} \mathrm{H}_{37} \mathrm{NO}_{3} \mathrm{C}$ 77.20; H 8.56. Found C 77.34; H 8.74.

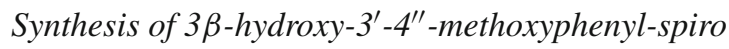
[androst-5-ene-16,5'S-2'-isoxazolin]-17 $\beta$-ol (12c)

Substrate: 11c (202 mg). 12c was obtained as a white solid $(197 \mathrm{mg}, 97 \%)$, mp $235-237{ }^{\circ} \mathrm{C}, R_{\mathrm{f}}=0.44$ (ss C); ${ }^{1} \mathrm{H}$ NMR (DMSO-d $6,500 \mathrm{MHz}): \delta_{\mathrm{H}} 0.66\left(\mathrm{~s}, 3 \mathrm{H}, 18-\mathrm{H}_{3}\right), 0.95(\mathrm{~s}, 3 \mathrm{H}$, 19- $\left.\mathrm{H}_{3}\right), 0.97(\mathrm{~m}, 1 \mathrm{H}), 1.14-1.27$ (overlapping $\mathrm{m}, 3 \mathrm{H}$ ), 1.311.43 (overlapping $\mathrm{m}, 3 \mathrm{H}$ ), 1.45-1.55 (overlapping $\mathrm{m}, 3 \mathrm{H}$ ), $1.67-1.78(\mathrm{~m}, 3 \mathrm{H}), 1.88(\mathrm{~m}, 1 \mathrm{H}), 1.97(\mathrm{~m}, 1 \mathrm{H}), 2.06-2.16$ (overlapping m, 2H), $2.96\left(\mathrm{~d}, 1 \mathrm{H}, J=17.1 \mathrm{~Hz}\right.$, one of $4^{\prime}-\mathrm{H}$ ), $3.26(\mathrm{~m}, 1 \mathrm{H}, 3-\mathrm{H}), 3.61(\mathrm{~d}, 1 \mathrm{H}, J=4.9 \mathrm{~Hz}, 17-\mathrm{H}), 3.72(\mathrm{~d}$, $1 \mathrm{H}, J=17.1 \mathrm{~Hz}$, the other $\left.4^{\prime}-\mathrm{H}\right), 3.78$ (s, 3H, $\left.4^{\prime \prime}-\mathrm{OMe}\right), 4.64$ $(\mathrm{d}, 1 \mathrm{H}, J=4.2 \mathrm{~Hz}, 3-\mathrm{OH}), 5.26(\mathrm{~m}, 1 \mathrm{H}, 6-\mathrm{H}), 5.31(\mathrm{~d}, 1 \mathrm{H}, J$ $=4.9 \mathrm{~Hz}, 17-\mathrm{OH}), 6.98\left(\mathrm{~d}, 2 \mathrm{H}, J=8.6 \mathrm{~Hz}, 3^{\prime \prime}-\mathrm{H}\right.$ and $\left.5^{\prime \prime}-\mathrm{H}\right)$, 7.59 (d, $2 \mathrm{H}, J=8.6 \mathrm{~Hz}, 2^{\prime \prime}-\mathrm{H}$ and $\left.6{ }^{\prime \prime}-\mathrm{H}\right) ;{ }^{13} \mathrm{C}$ NMR (DMSO$\left.\mathrm{d}_{6}, 125 \mathrm{MHz}\right): \delta_{C} 11.4(\mathrm{C}-18), 19.1(\mathrm{C}-19), 20.0\left(\mathrm{CH}_{2}\right), 30.8$ (CH), $30.9\left(\mathrm{CH}_{2}\right), 31.4\left(\mathrm{CH}_{2}\right), 36.2\left(\mathrm{CH}_{2}\right), 36.4(\mathrm{C}-10), 36.8$ $\left(\mathrm{CH}_{2}\right), 41.5\left(\mathrm{CH}_{2}\right), 41.7(\mathrm{C}-13), 42.2\left(\mathrm{CH}_{2}\right), 42.6\left(\mathrm{CH}_{2}\right)$, $47.7(\mathrm{CH}), 49.7(\mathrm{CH}), 55.2$ (4" -OMe), $69.9(\mathrm{C}-3), 85.0(\mathrm{C}-$ 17), 94.5 (C-16), 114.1 (2C, C-3" and C-5"), 120.1 (C-6), $122.4\left(\mathrm{C}-1^{\prime \prime}\right), 127.8$ (2C, C-2" and C-6") 141.3 (C-5), 156.0 
$\left(\mathrm{C}-3^{\prime}\right), 160.3\left(\mathrm{C}-4^{\prime \prime}\right)$; ESI-MS $475[\mathrm{M}+\mathrm{Na}]^{+}$; Anal. Calcd. for $\mathrm{C}_{28} \mathrm{H}_{37} \mathrm{NO}_{4} \mathrm{C} 74.47 ; \mathrm{H}$ 8.26. Found C 74.61; H 8.36.

Synthesis of $3 \beta$-hydroxy-3'-4" -chlorophenylspiro[androst-5-ene-16,5'S-2'-isoxazolin]-17ß-ol (12d)

Substrate: 11d (204 mg). 12d was obtained as a white solid (195 mg, $95 \%$ ), decomposed above $190^{\circ} \mathrm{C}, R_{\mathrm{f}}=0.48$ (ss C) ${ }^{1} \mathrm{H}$ NMR (DMSO- $\left.\mathrm{d}_{6}, 500 \mathrm{MHz}\right): \delta_{\mathrm{H}} 0.66\left(\mathrm{~s}, 3 \mathrm{H}, 18-\mathrm{H}_{3}\right)$, 0.95 (s, 3H, 19- $\left.\mathrm{H}_{3}\right), 1.00(\mathrm{~m}, 2 \mathrm{H}), 1.19(\mathrm{~m}, 2 \mathrm{H}), 1.31-1.43$ (overlapping $\mathrm{m}, 3 \mathrm{H}$ ), 1.49-1.55 (overlapping $\mathrm{m}, 3 \mathrm{H}$ ), 1.68 $(\mathrm{m}, 1 \mathrm{H}), 1.76(\mathrm{~m}, 2 \mathrm{H}), 1.88(\mathrm{~m}, 1 \mathrm{H}), 1.99(\mathrm{~m}, 1 \mathrm{H}), 2.06-$ 2.17 (overlapping m, 2H), $3.01(\mathrm{~d}, 1 \mathrm{H}, J=17.2 \mathrm{~Hz}$, one of $\left.4^{\prime}-\mathrm{H}\right), 3.26(\mathrm{~m}, 1 \mathrm{H}, 3-\mathrm{H}), 3.62(\mathrm{~d}, 1 \mathrm{H}, J=4.9 \mathrm{~Hz}, 17-\mathrm{H})$, $3.76\left(\mathrm{~d}, 1 \mathrm{H}, J=17.2 \mathrm{~Hz}\right.$, the other $\left.4^{\prime}-\mathrm{H}\right), 4.60(\mathrm{~d}, 1 \mathrm{H}, J$ $=4.4 \mathrm{~Hz}, 3-\mathrm{OH}), 5.25(\mathrm{~m}, 1 \mathrm{H}, 6-\mathrm{H}), 5.33(\mathrm{~d}, 1 \mathrm{H}, J=5.0$ $\mathrm{Hz}, 17-\mathrm{OH}), 7.49\left(\mathrm{~d}, 2 \mathrm{H}, J=8.4 \mathrm{~Hz}, 3^{\prime \prime}-\mathrm{H}\right.$ and $\left.5^{\prime \prime}-\mathrm{H}\right), 7.68$ (d, $2 \mathrm{H}, J=8.4 \mathrm{~Hz}, 2^{\prime \prime}-\mathrm{H}$ and $\left.66^{\prime \prime}-\mathrm{H}\right) ;{ }^{13} \mathrm{C}$ NMR (DMSO$\left.\mathrm{d}_{6}, 125 \mathrm{MHz}\right): \delta_{\mathrm{C}} 11.3(\mathrm{C}-18), 19.1(\mathrm{C}-19), 19.9\left(\mathrm{CH}_{2}\right), 30.7$ $(\mathrm{CH}), 30.9\left(\mathrm{CH}_{2}\right), 31.3\left(\mathrm{CH}_{2}\right), 36.1\left(\mathrm{CH}_{2}\right), 36.3(\mathrm{C}-10), 36.7$ $\left(\mathrm{CH}_{2}\right), 40.9\left(\mathrm{CH}_{2}\right), 41.5(\mathrm{C}-13), 42.1\left(\mathrm{CH}_{2}\right), 42.6\left(\mathrm{CH}_{2}\right)$, $47.6(\mathrm{CH}), 49.6(\mathrm{CH}), 69.8(\mathrm{C}-3), 85.0(\mathrm{C}-17), 95.3(\mathrm{C}-16)$, $120.0(\mathrm{C}-6), 127.7\left(\mathrm{C}-1^{\prime \prime}\right), 127.9\left(2 \mathrm{C}, \mathrm{C}-3^{\prime \prime}\right.$ and $\left.\mathrm{C}-5^{\prime \prime}\right), 128.7$ (2C, C-2" and C-6"), 134.1 (C-4"), 141.3 (C-5), 155.6 (C$3^{\prime}$ ); ESI-MS $479[\mathrm{M}+\mathrm{Na}]^{+}$; Anal. Calcd. for $\mathrm{C}_{27} \mathrm{H}_{34} \mathrm{ClNO}_{3}$ C 71.11; H 7.52. Found C 71.01; H 7.64.

Synthesis of $3 \beta$-hydroxy- $3^{\prime}-4^{\prime \prime}$-nitrophenylspiro[androst-5-ene-16,5'S-2'-isoxazolin]-17 $\beta$-ol (12e)

Substrate: 11e (209 mg). 12e was obtained as a pale-yellow solid (204 mg, $97 \%$ ), mp 271-274 ${ }^{\circ} \mathrm{C}, R_{\mathrm{f}}=0.49$ (ss C); ${ }^{1} \mathrm{H}$ NMR (DMSO-d $\left.6,500 \mathrm{MHz}\right): \delta_{\mathrm{H}} 0.67\left(\mathrm{~s}, 3 \mathrm{H}, 18-\mathrm{H}_{3}\right)$, $0.95\left(\mathrm{~s}, 3 \mathrm{H}, 19-\mathrm{H}_{3}\right), 0.99(\mathrm{~m}, 2 \mathrm{H}), 1.20(\mathrm{~m}, 2 \mathrm{H}), 1.31-1.43$ (overlapping $\mathrm{m}, 3 \mathrm{H}$ ), 1.50-1.58 (overlapping $\mathrm{m}, 3 \mathrm{H}$ ), 1.68 $(\mathrm{m}, 1 \mathrm{H}), 1.76(\mathrm{~m}, 2 \mathrm{H}), 1.88(\mathrm{~m}, 1 \mathrm{H}), 2.01-2.17$ (overlapping $\mathrm{m}, 3 \mathrm{H}), 3.11\left(\mathrm{~d}, 1 \mathrm{H}, J=17.3 \mathrm{~Hz}\right.$, one of $\left.4^{\prime}-\mathrm{H}\right), 3.27(\mathrm{~m}, 1 \mathrm{H}$, $3-\mathrm{H}), 3.64(\mathrm{~d}, 1 \mathrm{H}, J=4.8 \mathrm{~Hz}, 17-\mathrm{H}), 3.82(\mathrm{~d}, 1 \mathrm{H}, J=17.3 \mathrm{~Hz}$, the other $\left.4^{\prime}-\mathrm{H}\right), 4.60(\mathrm{~d}, 1 \mathrm{H}, J=4.5 \mathrm{~Hz}, 3-\mathrm{OH}), 5.26(\mathrm{~m}, 1 \mathrm{H}$, 6-H), 5.38 (d, 1H, $J=4.8 \mathrm{~Hz}, 17-\mathrm{OH}), 7.92(\mathrm{~d}, 2 \mathrm{H}, J=8.7$ $\mathrm{Hz}, 2^{\prime \prime}-\mathrm{H}$ and $\left.6^{\prime \prime}-\mathrm{H}\right), 8.27$ (d, 2H, $J=8.6 \mathrm{~Hz}, 3^{\prime \prime}-\mathrm{H}$ and $\left.5^{\prime \prime}-\mathrm{H}\right)$; ${ }^{13} \mathrm{C}$ NMR (DMSO-d $6,125 \mathrm{MHz}$ ): $\delta_{\mathrm{C}} 11.3$ (C-18), 19.1 (C19), $19.9\left(\mathrm{CH}_{2}\right), 30.7(\mathrm{CH}), 30.8\left(\mathrm{CH}_{2}\right), 31.3\left(\mathrm{CH}_{2}\right), 36.1$ $\left(\mathrm{CH}_{2}\right), 36.2(\mathrm{C}-10), 36.7\left(\mathrm{CH}_{2}\right), 40.5\left(\mathrm{CH}_{2}\right), 41.5(\mathrm{C}-13)$, $42.1\left(\mathrm{CH}_{2}\right), 42.6\left(\mathrm{CH}_{2}\right), 47.6(\mathrm{CH}), 49.6(\mathrm{CH}), 69.8(\mathrm{C}-3)$, 85.0 (C-17), 96.4 (C-16), 120.0 (C-6), 123.9 (2C, C-3" and C-5"), 127.3 (2C, C-2" and C-6"), $136.0\left(\mathrm{C}-1^{\prime \prime}\right), 141.3(\mathrm{C}-$ 5), $147.6\left(\mathrm{C}-4^{\prime \prime}\right), 155.4\left(\mathrm{C}-3^{\prime}\right)$; ESI-MS $490[\mathrm{M}+\mathrm{Na}]^{+}$; Anal. Calcd. for $\mathrm{C}_{27} \mathrm{H}_{34} \mathrm{~N}_{2} \mathrm{O}_{5} \mathrm{C}$ 69.50; H 7.35. Found C 69.64; H 7.54 .
Determination of antiproliferative activities

Antiproliferative effects were measured in vitro on three human cancer cell lines (ECACC; Salisbury, UK): HeLa (cervix adenocarcinoma), MCF7 (breast adenocarcinoma), and A431 (skin epidermoid carcinoma). The cells were cultivated in minimal essential medium (Sigma-Aldrich, Budapest, Hungary) supplemented with $10 \%$ fetal bovine serum, $1 \%$ non-essential amino acids, and an antibioticantimycotic mixture. Near-confluent cells were seeded into a 96-well plate (5,000 cells/well) and, after overnight standing, the medium $(200 \mu \mathrm{L})$ containing the tested compound (at 10 or $30 \mu \mathrm{M}$ ) was added. Following a 72 -h incubation period in a humidified atmosphere of $5 \% \mathrm{CO}_{2}$ at $37^{\circ} \mathrm{C}$, the living cells were assayed by the addition of $20 \mu \mathrm{L}$ of $5 \mathrm{mg} / \mathrm{mL}$ MTT [3-(4,5-dimethylthiazol-2-yl)-2,5-diphenyltetrazolium bromide] solution [44]. During a 4-h contact period, the MTT was converted by intact mitochondrial reductase and precipitated as blue crystals. The medium was then removed, the precipitated formazan crystals were solubilized in DMSO $(100 \mu \mathrm{L})$ during a 60 -min period of shaking at $25^{\circ} \mathrm{C}$, and the absorbance was read at $545 \mathrm{~nm}$ with a microplate reader. Wells with untreated cells were utilized as controls. All in vitro experiments were carried out on two microplates with at least five parallel wells. Stock solutions of the tested substances $(10 \mathrm{mM})$ were prepared with DMSO. The DMSO concentration $(0.3 \%)$ of the medium did not have any significant effect on cell proliferation. Cisplatin was used as a reference compound. A set of dilutions were additionally applied in order to determine the $\mathrm{IC}_{50}$ values of the most active compounds. Sigmoidal dose-response curves were fitted to the measured points, and the values were calculated by means of GraphPad Prism 4.0 (GraphPad Software; San Diego, CA, USA).

Acknowledgments The financial support by the Hungarian Scientific Research Fund (OTKA K-109107) and the New Hungary Development Plan (TÁMOP-4.2.2.A-11/1/KONV-2012-0047) is gratefully acknowledged. The research by D. Kovács was supported by the European Union and the State of Hungary, co-financed by the European Social Fund in the framework of TÁMOP-4.2.4.A/2-11/1-2012-0001 "National Excellence Program".

\section{References}

1. Stulov SV, Misharin AYu (2013) Synthesis of steroids with nitrogen-containing substituents in ring D (Review). Chem Heterocycl Comp 48:1431-1472. doi:10.1007/s10593-013-1158-8

2. Frank É, Schneider Gy (2013) Synthesis of sex hormone-derived modified steroids possessing antiproliferative activity. J Steroid Biochem Mol Biol 137:301-315. doi:10.1016/j.jsbmb.2013.02. 018

3. Singh R, Panda G (2013) An overview of synthetic approaches for heterocyclic steroids. Tetrahedron 69:2853-2884. doi:10.1016/j. tet.2013.02.018 
4. Munari CC, de Oliveira PF, Campos JC, Martins SD, Da Costa JC, Bastos JK, Tavares DC (2014) Antiproliferative activity of Solanum lycocarpum alkaloidic extract and their constituents, solamargine and solasodine, in tumor cell lines. J Nat Med 68:236-241. doi:10. 1007/s11418-013-0757-0

5. Friedman M, Levin CE, Lee S-U, Kim H-J, Lee I-S, Byun JO, Kozokue N (2009) Tomatine-containing green tomato extracts inhibit growth of human breast, colon, liver, and stomach cancer cells. J Agric Food Chem 57:5727-5733. doi:10.1021/ jf900364j

6. Tietze LF, Scneider Gy, Wölfling J, Fecher A, Nöbel T, Petersen S, Schuberth I, Wulff C (2000) A novel approach in drug discovery: synthesis of estrone-talaromycin natural product hybrids. Chem-Eur J 6:3755-3760. doi:10.1002/1521-3765(20001016)6: 20<3755:AID-CHEM3755>3.0.CO;2-L

7. Tietze LF (1998) Schneider Gy, Wölfling J, Nöbel T, Wulff C, Schuberth I, Rübeling A Efficient synthesis of a novel Estrone-Talaromycin hybrid natural product. Angew Chem Int Ed 37:2469-2470. doi:10.1002/(SICI)1521-3773(19981002)37: 18<2469:AID-ANIE2469>3.0.CO;2-M

8. Pradhan R, Patra M, Behera AK, Mishra BK, Behera RK (2006) A synthon approach to spiro compounds. Tetrahedron 62:779-828. doi:10.1016/j.tet.2005.09.039

9. Allous I, Comesse S, Sanselme M, Daïch A (2011) Diastereoselective access to tri- and pentacyclic spiro- $\gamma$-lactam-oxindole cores through a tandem aza-Michael initiated ring closure sequence. Eur J Org Chem 5303-5310: doi:10.1002/ejoc.201100731

10. Hilton ST, Ho TCT, Pljevaljcic G, Jones K (2000) A new route to spirooxindoles. Org Lett 2:2639-2640. doi:10.1021/ ol0061642

11. Kanchithalaivan S, Kumar RR, Perumal S (2013) Synthesis of novel 16-spiro steroids: spiro- $7^{\prime}$-(aryl)tetrahydro- $1 H$-pyrrolo[1,2c] $[1,3]$ thiazolo-trans-androsterone hybride heterocycles. Steroids 78:409-417. doi:10.1016/j.steroids.2012.12.017

12. Rapi G, Ginanneschi M, Chelli M, Chimichi S (1985) Reaction os some antiinflammatory $17 \beta$-(2-aminooxazol-4-yl) steroids with hydrogen peroxide. Synthesis of steroid-17-spiro- $5^{\prime}$ oxalidine-2',4'-diones. Steroids 46:665-676. doi:10.1016/0039128X(85)90030-3

13. Mernyák E, Kozma E, Hetényi A, Márk L, Schneider Gy, Wölfling J (2009) Stereoselective synthesis of spiro and condensed pyrazolines of steroidal $\alpha, \beta$-saturated ketones and nitrilimines by 1,3-dipolar cycloaddition. Steroids 74:520-525. doi:10.1016/j. steroids.2009.02.001

14. Babu ARS, Raghunathan R (2008) An easy access to novel steroidal dispiropyrrolidines through 1,3-dipolar cycloaddition of azomethine ylides. Tetrahedron Lett 49:4618-4620. doi:10.1016/j.tetlet. 2008.05.089

15. Frank É, Sipos L, Wölfling J, Schneider Gy (2009) Synthesis and conformational preferences of novel steroidal 16-spiro-1,3,2dioxaphosphorinanes. Lett Org Chem 6:340-344. doi:10.2174/ 157017809788489927

16. Wölfling J, Kovács-Pénzes P, Zupkó I, Schneider Gy, Frank É (2012) Synthesis, stereochemistry and cytotoxic activity of novel steroidal 16-spiro-1,3,2-dioxaphosphorinanes. J Mol Struct 1013:39-44. doi:10.1016/j.molstruc.2012.01.013

17. Mohamed NR, Elmegeed GA, Abd-ElMalek HA, Younis M (2005) Synthesis of biologically active steroid derivatives by the utility of Lawesson's reagent. Steroids 70:131-136. doi:10.1016/j.steroids. 2004.11.001

18. Krstić NM, Bjelaković MS, Pavlović VD, Robeyns K, Juranić ZD, Matić I, Novaković I, Sladić DM (2012) New androst-4en-17-spiro-1,3,2-oxathiaphospholanes. Synthesis, assignment of absolute configuration and in vitro cytotoxic and antimicrobal activities. Steroids 77:558-565. doi:10.1016/j.steroids.2012.01. 021
19. Drach SV, Litvinovskaya RP, Khripach VA (2000) Steroidal 1,2oxazoles. Synthesis and biological activity (Review). Chem Heterocycl Compds 36:233-255. doi:10.1007/BF02256860

20. Peng J, Li J, Hamann MT (2005) The marine bromotyrosine derivatives. Alkaloids Chem Biol 61:59-262. doi:10.1016/S10994831(05)61002-4

21. Jäger $V$, Grund $H$ (1976) Eliminative ring opening of 2-Isoxazolines: A new route to $\alpha, \beta$-unsaturated ketones. Angew Chem Int Ed 15:50-51. doi:10.1002/anie.197600501

22. Curren DP (1982) Reduction of $\Delta^{2}$-isoxazolines: a conceptually different approach to the formation of aldol adducts. J Am Chem Soc 104:4024-4026. doi:10.1021/ja00378a050

23. Jäger V, Buss V, Schwab W (1978) Syntheses via isoxazolines III. Diastereoselective synthesis of $\gamma$-amino-alcohols with 2 and 3 chiral centres. Tetrahedron Lett 19:3133-3136. doi:10.1016/S00404039(01)94963-9

24. Rahbœk L, Christophersen C (2001) The isoxazole alkaloids. Alkaloids Chem Biol 57:185-262. doi:10.1016/S0099-9598(01)570042

25. Pohjakallio A (2011) Synthesis and reactions of 3-unsubstituted 2-isoxazolines. Dissertation, Aalto University

26. Camoutsis Ch, Nikolaropoulos S (1998) Steroidal isoxazoles, isoxazolines and isoxazolidines. J Heterocycl Chem 35:731-759. doi:10.1002/jhet.5570350401

27. Jäger V, Colinas PA (2003) Nitrile oxides. In: Padwa A, Pearson WH (eds) Synthetic applications of 1,3-dipolar cycloaddition chemistry toward heterocycles and natural products, John Wiley, Hoboken, Chapter 6, pp 361-472

28. Huisgen R (1984) 1,3-Dipolar cycloadditions-introduction, survey, mechanism. In: Padwa, A (ed) 1,3-Dipolar Cycloaddition Chemistry, John Wiley, New York, vol 1, Chapter 1, pp 1-176

29. Frank É, Mucsi Z, Szécsi M, Zupkó I, Wölfling J, Schneider Gy (2010) Intramolecular approach to some new D-ring-fused steroidal isoxazolidines by 1,3-dipolar cycloaddition: synthesis, theoretical and in vitro pharmacological studies. New J Chem 34:2671-2681. doi:10.1039/c0nj00150c

30. Frank É, Mucsi Z, Zupkó I, Réthy B, Falkay G, Schneider Gy, Wölfling J (2009) Efficient approach to androstene-fused arylpyrazolines as potent antiproliferative agents. Experimental and theoretical studies of substituent effects on $\mathrm{BF}_{3}$-catalyzed intramolecular [3+2] cycloadditions of olefinic phenylhydrazones. J Am Chem Soc 131:3894-3904. doi:10.1021/ja808636e

31. Kádár Z, Baji Á, Zupkó I, Bartók T, Wölfling J, Frank É (2011) Efficient approach to novel $1 \alpha$-triazolyl- $5 \alpha$-androstane derivatives as potent antiproliferative agents. Organ Biomol Chem 9:80518057. doi:10.1039/c1ob06086d

32. Kovács D, Kádár Z, Mótyán G, Gy. Schneider, J. Wölfling, I. Zupkó, É. Frank, (2012) Synthesis, characterization and biological evaluation of some novel 17-isoxazoles in the estrone series. Steroids 77:1075-1085. doi:10.1016/j.steroids.2012.05.003

33. Bakos T, Vincze I (1992) A new route to 16-methylene17-ketosteroids. Synth Commun 22:1377-1383. doi:10.1080/ 00397919208021602

34. Schneider Gy, Vincze I, Hackler L, Dombi Gy (1983) A convenient method for the formation of 16-methylene-17-ketosteroids. Synthesis 665-669: doi:10.1055/s-1983-30466

35. Caramella P, Grünanger P (1984) Nitrile oxides and imines. In: Padwa, A (ed) 1,3-Dipolar cycloaddition chemistry, John Wiley, New York, vol 1, Chapter 3, p 326

36. Himo F, Lovell T, Hilgraf R, Rostovtsev VV, Noodleman L, Sharpless KB, Fokin VV (2005) Copper(I)-catalyzed synthesis of azoles. DFT study predicts unprecedented reactivity and intermediates. J Am Chem Soc 127:210-216. doi:10.1021/ja0471525

37. Liu KC, Shelton BR, Howe RK (1980) A particularly convenient preparation of benzo- hydroximinoyl chlorides (nitrile oxide precursors). J Org Chem 45:3916-3918. doi:10.1021/jo01307a039 
38. Grundmann C, Grünanger P (1971) The nitrile oxides. Springer, Berlin

39. Grundmann C (1970) Synthesis of heterocyclic compounds with the aid of nitrile oxides. Synthesis 344-359: doi:10.1055/s-197021611

40. Savage GP (2010) Spiro isoxazolines via nitrile oxide 1,3-dipolar cycloaddition reactions. Curr Organ Chem 14:1478-1499. doi:10. 2174/138527210791616812

41. Khazir J, Singh PP, Reddy DM, Hyder I, Shafi S, Sawant SD et al (2013) Synthesis and anticancer activity of novel spiro-isoxazoline and spiro-isoxazolidine derivatives of $\alpha$-santonin. Eur J Med Chem 63:279-289. doi:10.1016/j.ejmech.2013.01.003
42. Najim N, Bathich Y, Zain MM, Hamzah AS, Shaameri Z (2010) Evaluation of the bioactivity of novel spiroisoxazoline type compounds against normal and cancer cell lines. Molecules 15:93409353. doi:10.3390/molecules 15129340

43. Mosmann T (1983) Rapid colorimetric assay for cellular growth and survival: application to proliferation and cytotoxicity assays. J Immunol Methods 65:55-63. doi:10.1016/0022-1759(83)90303-4 